\title{
The crosstalk between reactive oxygen species and noncoding RNAs: from cancer code to drug role
}

\author{
Jing Zuo ${ }^{1 \dagger}$, Zhe Zhang ${ }^{1 \dagger}$, Maomao $\mathrm{Li}^{2 \dagger}$, Yun Yang ${ }^{2 \dagger}$, Bohao Zheng ${ }^{2}$, Ping Wang ${ }^{2 *}$, Canhua Huang ${ }^{1 *}$ and \\ Shengtao Zhou ${ }^{2^{*}}$ (D)
}

\begin{abstract}
Oxidative stress (OS), characterized by the excessive accumulation of reactive oxygen species (ROS), is an emerging hallmark of cancer. Tumorigenesis and development driven by ROS require an aberrant redox homeostasis, that activates onco-signaling and avoids ROS-induced programmed death by orchestrating antioxidant systems. These processes are revealed to closely associate with noncoding RNAs (ncRNAs). On the basis of the available evidence, ncRNAs have been widely identified as multifarious modulators with the involvement of several key redox sensing pathways, such as NF-KB and Nrf2 signaling, therefore potentially becoming effective targets for cancer therapy. Furthermore, the vast majority of ncRNAs with property of easy detected in fluid samples (e.g., blood and urine) facilitate clinicians to monitor redox homeostasis, indicating a novel method for cancer diagnosis. Herein, focusing on carcinoma initiation, metastasis and chemoradiotherapy resistance, we aimed to discuss the ncRNAs-ROS network involved in cancer progression, and the potential clinical application as biomarkers and therapeutic targets.
\end{abstract}

Keywords: Reactive oxygen species, Oxidative stress, Noncoding RNAs, Cancer diagnosis, Cancer therapy

\section{Introduction}

Intracellular oxidative reactions play a vital role in metabolic processes, by which plenty of biomolecular compounds like reactive species are produced. Under normal physiological conditions, these products maintain proper concentrations, with a close relation to enzyme activation $[1-3]$, protein synthesis $[4,5]$, signal transduction $[6,7]$

\footnotetext{
*Correspondence: wangping_886@126.com; hcanhua@scu.edu.cn; taotaovip2005@163.com

†Jing Zuo, Zhe Zhang, Maomao Li and Yun Yang contributed equally to this work.

${ }^{1}$ State Key Laboratory of Biotherapy and Cancer Center, West China Hospital, and West China School of Basic Medical Sciences \& Forensic Medicine, Sichuan University, and Collaborative Innovation Center for Biotherapy, Chengdu 610041, People's Republic of China

${ }^{2}$ Department of Obstetrics and Gynecology, Key Laboratory of Birth Defects and Related Diseases of Women and Children of MOE and State Key Laboratory of Biotherapy, West China Second University Hospital, Sichuan University and Collaborative Innovation Center, Chengdu, People's Republic of China
}

and gene expression $[8,9]$. However, excessive products have some detrimental impacts, probably leading to the dysregulation of the above biological events and causing all different kinds of diseases, including inflammation [10], diabetes [11], cardiovascular disease [12] and even malignancies [13]. Therefore, the maintenance of redox homeostasis is essential to normal cellular activities and human health. There are four main reactive species: reactive oxygen species (ROS), reactive nitrogen species (RNS), reactive sulfur species (RSS) and reactive chlorine species (RCS). ROS is the most abundantly produced and frequently explored, involving superoxide anion (O2-), hydrogen peroxide $\left(\mathrm{H}_{2} \mathrm{O}_{2}\right)$, hydroxyl radical $(\mathrm{OH}-)$, singlet oxygen (1O2) and ozone (O3) [14]. ROS originates from internal oxygen metabolism and external environmental changes. Internal sources stem from oxygen, which is catalyzed by different enzymes, and primarily occurs in mitochondria, peroxisomes and endoplasmic 
reticulum (ER) [15] . Meanwhile, external inducers are composed of ultraviolet radiation, ionizing radiation and toxic compounds, which generate accumulated ROS and probably cause cancerous transformation [16-18].

OS refers to the equilibrium disturbance of oxidative and anti-oxidative systems in favor of oxidant burden, which is generally mediated by ROS. Normally, intracellular antioxidants are enough to neutralize extra oxides to maintain homeostasis and ensure a balanced status. However, when redox balance is broken, extra ROS will induce $O S$ and damage biomacromolecules, including DNA [19-21], RNA [22, 23], proteins [24] and lipids [25-27], engendering cell death or provoking the malignant transformation of normal cells. Intriguingly, excessive ROS seems to preferentially accumulate in cancer cells. Thus, there are two interesting issues worthy of discussion: how cancer cells protect themselves from ROS toxicity and whether ROS-based anticancer strategies benefit cancer therapy.

NcRNAs are defined as transcripts that cannot be translated into proteins or functional proteins at least. In recent years, ncRNAs have attracted much interest and been proved to be direct or indirect elements of gene transcriptional and post-transcriptional regulation [28-31]. A large number of ncRNAs have been identified, which are classified into two major groups in length: short ncRNAs $(<200 \mathrm{bp})$ and long ncRNAs (lncRNAs) (>200 bp). Short ncRNAs mainly comprise microRNAs (miRNAs), circular RNAs (circRNAs), small interfering RNAs (siRNAs), small nuclear RNAs (snRNAs), small nucleolar RNAs (snoRNAs), PIWI-interacting RNAs (piRNAs), tRNA-derived small RNAs (tsRNAs) and enhancer noncoding RNAs (eRNAs) [32, 33]. A growing number of ncRNAs are regarded as possible diagnostic and prognostic biomarkers of cancer dynamics and treatment monitoring, for instance, circulating U2 small nuclear RNA functioned as a biomarker in epithelial ovarian cancer and lymphoma [34, 35]. Furthermore, considering the widespread functions of ncRNAs in cancer, the enormous therapeutic potential of ncRNAs is nonnegligible. siRNAs possess the advantages of smaller size, targetable features and simplex working principle, making it possible to treat cancer by silencing key oncogenes with the extensive application of nanotechnology $[36,37]$. Additionally, the interplay of ncRNAs with ROS has also been proved extensively, thereby a considerable treatment method referring to ncRNAs could be built on the basis of ROS. CircRNA-101,036, identified as a tumor suppressor, represses cancer development by inducing ER stress and substantial ROS accumulation in oral squamous cell carcinoma [38]. These results reveal that ncRNAs can become promising therapeutic targets and agents based on ROS.
For many years, most studies have been addicted to the drivers of cancer initiation and progression, such as aberrant expression of oncogenes and tumor suppressors or dysregulation of pivotal signaling pathways. They are ever considered as promising targets for cancer therapy, and some pharmaceutical companies have developed drugs based on these targets. Nevertheless, severe side-effects, low sensitivity and therapeutic resistance greatly limit their further application. Cancer cells have many unique characteristics compared with normal cells, including immortalization, metastasis, aerobic glycolysis, gene mutation and immune evasion. Targeting these characteristics may provide more optional treatment methods to kill cancer cells while sparing normal cells. OS is one of the most significant hallmarks within cancer cells, and has been reported to influence ncRNAs. NcRNAs can also modulate ROS in many ways, so profoundly understanding the crosstalk between ROS and ncRNAs will facilitate the development of new ROS-based ncRNAtargeted or ncRNA medicine. Herein, we systematically review the progress of ncRNAs and ROS interacting with each other in cancer mainly from a therapeutic perspective, emphasizing the potential of ncRNA-based agents in clinical application.

\section{The production and therapeutic potential of ROS in cancer}

ROS is primarily derived from mitochondria, whose oxidative metabolism efficiently produces ATPs to meet the energy demand of normal cells in the presence of oxygen. As a result of oxidative metabolism, the reactive species byproducts are generated, such as ROS. However, when mitochondrial dysregulation occurs, high ROS levels will release a ROS burst, causing mitochondrial destruction and even damaging the whole cell [39]. Generally, the antioxidant system can neutralize excessive ROS to prevent oxidative damage. While in cancer cells, elevated levels of ROS are commonly found, hence, it is confusing why cancer cells produce extra ROS and how they easily escape ROS damage and keep their malignant features of rapid proliferation, migration, invasion and apoptotic inhibition.

Metabolic stress, persistently occurs in tumor microenvironments, which implies a lack of nutrients, oxygen and growth factors because of excessive consumption via continuous proliferation and relatively insufficient angiogenesis of cancer cells [40], is a cause of ROS overload. Glucose deprivation of cancer cells directly leads to glycolysis blockade and ATP synthesis reduction, resulting in glycolysis-relevant antioxidant deficiency and provoking oxidative metabolism reoccurrence. Under such conditions, ROS production accelerates and elimination decreases, directly contributing to ROS accumulation. 
Nevertheless, ROS levels within cancer cells can be modulated to a proper degree through their robust antioxidant systems to utilize oncogenic roles. Cancer cells exhibit a powerful antioxidant system consisting of reductants such as the antioxidant enzymes-superoxide dismutase (SOD), catalase (CAT), glutathione peroxidase (GPX) and the antioxidant agents-nicotinamide adenine dinucleotide phosphate (NADPH) and glutathione (GSH). Antioxidants sustain a moderate ROS level to protect cells from ROS attack and facilitate cancer progression. Antioxidant production is regulated by multiple signaling pathways and genes, such as nuclear factor erythroid 2-related factor 2 (Nrf2) [41]. Moderate ROS levels can decrease the toxicity of natural killer cells (NK cells) [42] and increase the self-renewal capacity of cancer cells. In addition, it can also activate multiple protumorigenic signaling pathways such as NF- $\mathrm{BB}$, TGF- $\beta$, JAK2-STAT1 and PI3K/Akt/ERK or activate oncogenes and inhibit tumor suppressors to enhance cancer progression [43-47]. Accordingly, through above intracellular self-adaptation alterations, ROS will be partially counteracted and cancer cells survive even can be further promoted.

There should be a threshold value for ROS levels in regulating cell fate - malignant transformation and death, which may suggest underlying treatment regimens
(Fig. 1). Normal cells, cancer cells and cancer stem cells (CSCs) have different intracellular ROS loads that may be the basis of ROS-elevated cancer therapy [48]. A higher level of ROS prompts a lower dose demand of ROS-elevated drugs/radiation in killing cancer cells thus, this is a complicated and significant course to explore appropriate therapeutic doses and ensure treatment benefits. While in most cases, patients who accept traditional chemo/ radiotherapy show different side effects, including infections, anemia, fever, gastrointestinal discomfort and hair loss [49]. These impacts are probably derived from excessive treatments that cause ROS levels in normal cells to reach a toxic threshold and damage normal tissues. Moreover, decreasing treatment doses has difficulty killing most cancer cells and leads to cancer relapse. Additionally, CSCs have been universally acknowledged to be a pivotal element of cancer relapse. Stemness is a tough challenge for cancer therapy due to a lower ROS level in CSCs than that in normal cells.

In conclusion, although it shows great potential in cancer therapy, severe side effects are equally harmful. Hence, other replaceable therapeutic strategies on the foundation of ROS are urgently developed. Notably, ROS-based ncRNA-targeted or ncRNA medicine have exhibited some impressive achievements in cancer therapy, thus deeply understanding the crosstalk between

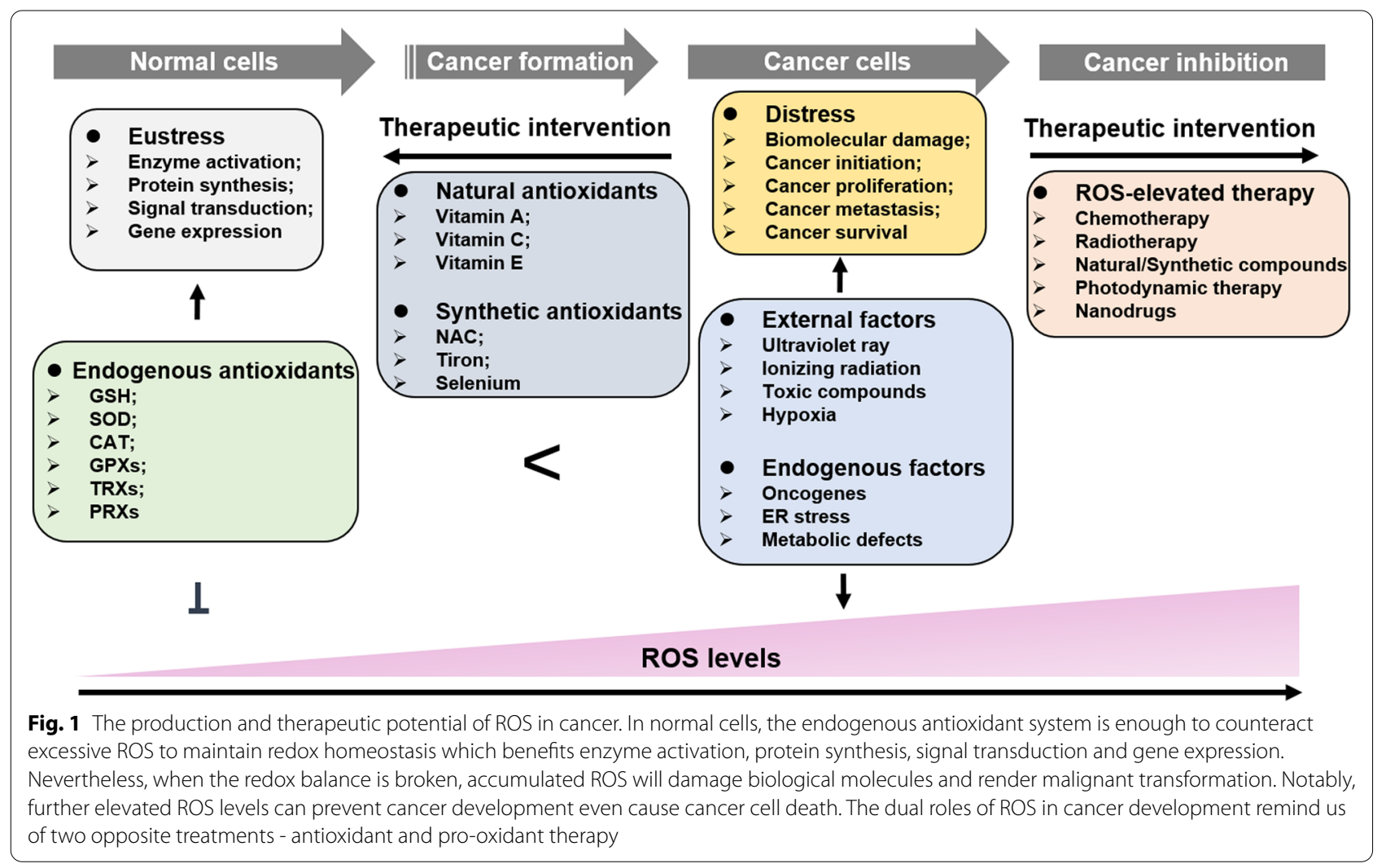


ROS and ncRNAs will greatly promote the development of ROS-based agents and benefit cancer patients.

\section{The mechanisms of ROS-ncRNAs axis in cancer progression}

Oxidative stress, that induces oxidative damage of biomacromolecules and aberrant modulation of redox signaling, is a key biological event throughout cancer initiation and development [50-52]. ROS as a second messenger during the moderate OS supports dysregulation of various pro-tumorigenic signaling pathways for malignant disease, in contrast, induction of excessive OS leads to cancer cell death by triggering ROS-driven apoptosis and ferroptosis $[53,54]$, thus indicating a potential development of OS-dependent strategy in clinical treatment. Accordingly, understanding the exquisite mechanism of redox modulation patterns facilitates a better therapeutic purpose by breaking redox homeostasis in cancer cells. NcRNAs occupy a majority of all transcripts, and are defined as dark matters in gene transcription at first. With in-depth investigation on the role of ncRNAs, they are commonly identified as being significantly involved in modulating carcinoma development [55-58], therefore displaying a promising potential as biomarkers or targets in cancer diagnosis and therapy [34, 35, 59, 60]. In this section, focusing on the detailed mechanism of the crosstalk between ncRNAs and ROS, we summarize the progress in recent years of ncRNAs involved in cancer initiation, metastasis and chemoradiotherapy resistance based on redox signaling regulation.

\section{Cancer initiation}

Cancer initiation, featuring uncontrollable cell proliferation and growth, is the best stage for cancer prevention. Carcinogenic environmental factors with ROS induction properties have been proved to be one of the main causes in many cancer patients [61, 62]. A growing body of studies has revealed the underlying mechanisms associated with ncRNAs-ROS axis-driven cancer initiation. For example, hexavalent chromium and arsenic were reported to increase ROS-dependent miR-21 transcription and compromise the expression of programmed cell death 4 (PDCD4), thus inducing the upregulation of downstream genes like E-cad, c-myc and uPAR and subsequently malignant transformation of bronchial epithelial cells $[16,63]$. Furthermore, a previous study indicated that ionizing radiation (IR)-induced malignant transformation resulted in excessive OS by miR-21-mediated downregulation of the antioxidant enzyme superoxide dismutase 2 (SOD2) [17]. In line with this, UVB-triggered cancer initiation was revealed to hold a close relationship with hexavalent chromium and arsenic-derived OS based on modulation of miR-21 [18]. In addition, bronchial epithelial cells under chronic arsenic exposure were found to have accelerated cancerization, which was attributed to high ROS levels promoted the expression of hypoxia-inducible factor- $1 \alpha$ (HIF-1 $\alpha$ ) and cyclooxygenases-2 (COX-2) by mitigating $m i R-199 a-5 p$ transcription [64].

In addition to miRNAs, lncRNAs involved in redox regulation also function in cancer initiation. The c-mycdownregulated lncRNA transcript IDH1-AS1 rendered the homodimerization of IDH1 and enhanced its enzymatic activity, which directly caused $\alpha-K G$ accumulation and OS and glycolysis blockage, preventing cancer proliferation at an early stage [65]. ROS-dependent lncRNA AX800134 was upregulated by TNF- $\alpha$ in hepatocellular carcinoma (HCC), which was elucidated to be responsible for the growth of cancer cells [66]. MACC1-AS1, an antisense of MACC1, was found to be increased in gastric cancer (GC). MACC1-AS1 maintained redox balance by stabilizing MACC1 mRNA and enhancing metabolic plasticity to promote the proliferation of cancer cells, which was indicated to be involved in AMPK/Lin28 signaling [67]. In melanoma, Wei et al found that lncRNA growth arrest-specific transcript 5 (GAS5) was significantly downregulated. The reduced transcription of GAS5 greatly alleviated oxidative stress and accelerated cell cycle progression through EZH2/CDKN1C axis [68].

Although miRNAs and lncRNAs dominate in OSmediated cancer initiation based upon available reports (Fig. 2), other ncRNAs still remain a large potential in the process. Although few studies have reported their interaction with ROS in cancer initiation, circRNAs have also been largely found to interact with ROS in other diseases and exhibit a promising role in cancer initiation. For instance, circRNA-101,036, identified as a tumor suppressor, induced ER stress and redox imbalance in oral squamous cell carcinoma [38]. As a sponge of miR-330-5p, tumor-suppressive human circular RNA $(\mathrm{CircITCH})$ significantly reduced $\mathrm{OS}$ and alleviated doxorubicin-induced cardiotoxicity via elevating the expression levels of SIRT6, Survivin and SERCA2a [69]. Likewise, in intestinal ischemia/reperfusion, circ-PRKCB was reported to sponge endogenous miR-339-5p, modulating p66Shc expression and redox signaling [70].

\section{Cancer metastasis}

Metastasis is one of the biggest obstacles in the treatment of terminal cancer patients. Understanding the detailed mechanisms in cancer metastasis may benefit the discovery of valuable therapeutic targets and the development of efficient interventions. Similarly, along with ROS, multiple types of ncRNAs have been confirmed to participate in cancer metastasis judging from current evidence (Fig. 3). 


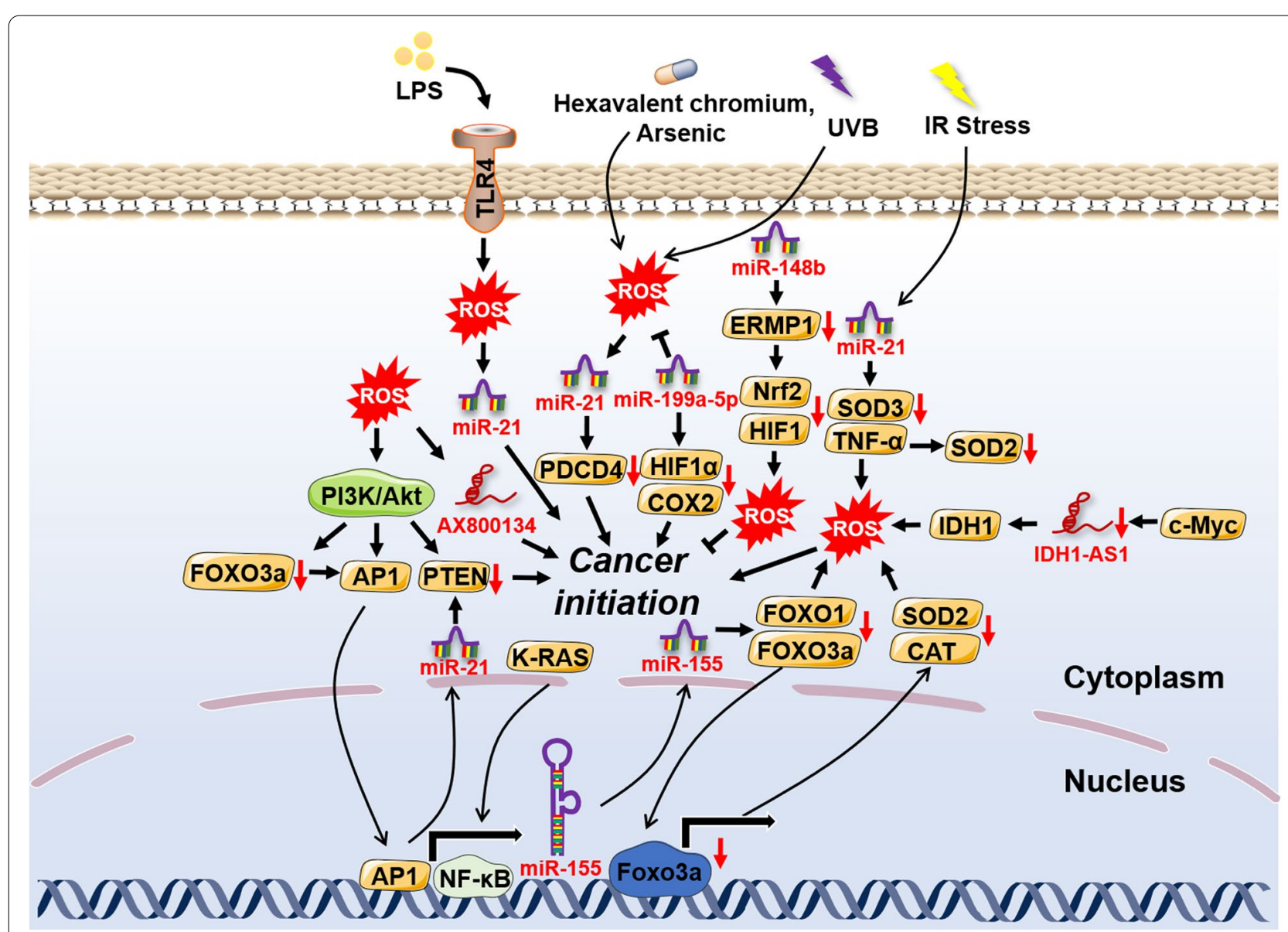

Fig. 2 The crosstalk of ROS with ncRNAs in cancer initiation. The external factors, such as ultraviolet ray, ionizing radiation and toxic compounds, and internal factors caused by the aberrant gene regulation can both lead to cancer initiation through the complex interplay of ROS with ncRNAs

Epithelial-mesenchymal transition (EMT), characterized by the transition from epithelial cells to cells with a mesenchymal $(\mathrm{M})$ phenotype, is a prerequisite of the metastasis for most solid cancers. EMT is triggered by some EMT-activating transcription factors (EMT-TFs), mainly of the SNAIL, TWIST and ZEB families and their targets like metalloproteinase-9 (MMP-9) [71]. During breast cancer progression, miR-373 quenched ROS levels by downregulating the ROS inducer thioredoxininteracting protein (TXNIP), stimulating HIF- $1 \alpha$ and TWIST expression [72]. Notably, TWIST bound to the promoter of the miR-371/373 cluster, which remarkably enhanced miR-373 transcription. Hence, the miR-373TXNIP-ROS-HIF $1 \alpha$-TWIST positive feedback signaling axis showed a powerful EMT-activating capacity. MiR$125 \mathrm{~b}$ was found to be downregulated in HCC tissues, which prompted mitochondrial protein $18 \mathrm{kDa}$ (MTP18) expression, leading to mitochondrial fission. Mitochondrial damage caused much ROS accumulation and increased MMP-9 expression, directly promoting EMT and cancer metastasis [73]. MiR-206 was reported to be modulated by nuclear factor NRF2 to drive tumorigenesis [74]. Simultaneously, miR-206 blocked TGF- $\beta$ signaling and downstream neuropilin-1 (NRP1) and smad2 expression, inhibiting EMT, migration and invasion of breast cancer cells [75]. Oncogenic miR-21 level was reduced via kallistatin protein-mediated ROS downregulation, which further suppressed Akt signaling and EMT in cancer cells [76].

In addition to miRNAs, mounting evidence indicates that lncRNAs are also engaged in cancer metastasis. LncRNA UCA1 worked as a ceRNA, which prevented the effects of miR-1 and miR-203a on Slug expression, promoting EMT and invasion in breast cancer [77]. Similarly, lncRNA-MUF (mesenchymal stem cell-upregulated factor) can also act as a ceRNA of miR-34a, activating Snail1 expression and $\mathrm{Wnt} / \beta$-catenin signaling to facilitate HCC metastasis [78]. Lnc-SNHG1 sponged miR$302 / 372 / 373 / 520$ to enhance the expression of target genes-TGF- $\beta$ receptor 2 (TGFBR2) and RAB11A, thus contributing to EMT in pituitary cancer [79]. Although the underlying mechanism remains unclear, we speculate 


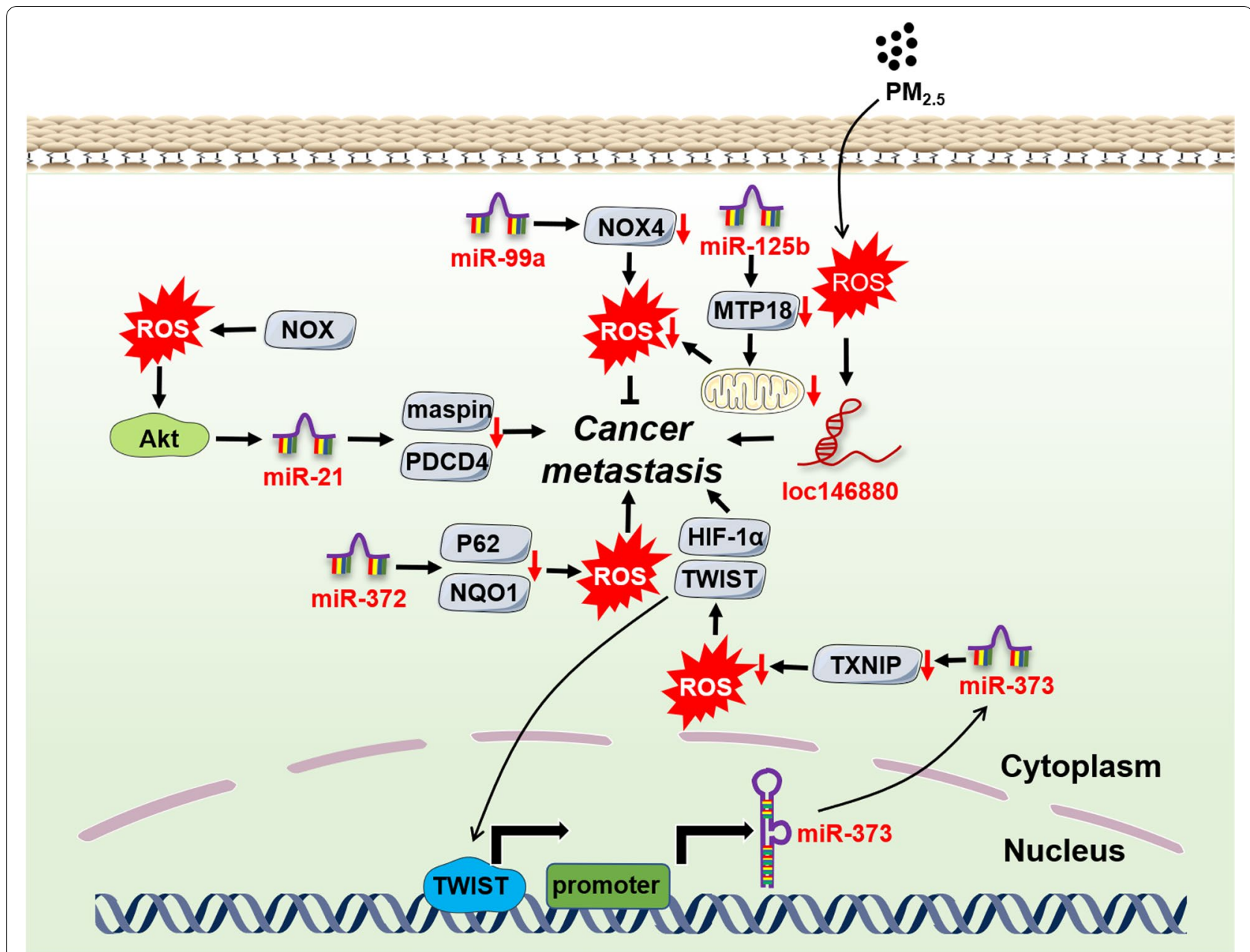

Fig. 3 The crosstalk of ROS with ncRNAs in cancer metastasis. Cancer metastasis is tightly regulated by the intricate interaction of ROS with ncRNAs which involved multiple pivotal molecules and signaling pathways

that it is related to miR-1/34a/372-triggered ROS regulation from available reports [74, 80, 81]. LncRNA MALAT1 was found to upregulate ROS levels via keap1/ Nrf1/2 signaling [82], which induced EMT and metastasis in head and neck squamous cell carcinoma (HNSCC) through STAT3 activation [83]. PM2.5 exposure of lung cancer cells significantly increased ROS levels and upregulated subsequent IncRNA loc146880 expression, causing malignant metastasis of lung cancer cells [84].

Besides miRNAs and LncRNAs, current studies have implied circRNAs are also capable of participating in OS-mediated cancer metastasis. CircSCAF11 overexpression inhibited ROS production by acting as a sponge of miR-145-5p in glioma, which is a cause of glioma initiation and metastasis [85]. MiR-30c-5p was reported to be an important OS regulator by multiple studies [86-88]. In colorectal cancer, circ3823 functioned as a ceRNA of miR-30c-5p, alleviating the repressive effect of miR-30c-5p on TCF7 which upregulated MYC and CCND1 and eventually led to cancer initiation and metastasis [89]. Hu et al identified an oncogenic circASAP1 through circRNA sequencing in patients with $\mathrm{HCC}$, which was proved to be associated with cancer initiation and metastasis via miR-326/miR-532-5p-MAPK1/ CSF-1 signaling, respectively [90]. The result suggested that the underlying mechanism was also modulated by OS $[91,92]$.

\section{Cancer chemoradiotherapy resistance}

Chemoradiotherapy resistance is the leading threat for cancer patients. Reversing chemoradiotherapy resistance is a pivotal bottleneck problem to be resolved. OS-induced chemoradiotherapy resistance has been commonly identified, targeting which will be conducive to overcoming multidrug resistance (MDR) and radiotherapy failure [93]. 
Increasing evidence indicates that CSCs are at top and responsible for cancer chemoradiotherapy resistance, relapse and treatment failure [94]. It was reported that CSCs possessed a lower ROS level, which may be exploited to develop ROS-elevated treatments to reserve CSC-mediated chemoradiotherapy resistance. As a target of miR-223, the inhibition of HAX-1 led to mitochondrial damage and accelerated ROS generation in triple-negative breast cancer stem cells (TNBCSCs), which ultimately enhanced TRAIL-induced apoptosis [95]. MiR-153 downregulated Nrf2 and GPX1 expression, by which the elevated ROS levels decreased the stemness of glioma stem cells (GSCs) and relieved radiation resistance [96]. However, Yang et al reported that the decreased ROS levels resulting from miR-210 were able to weaken the stemness of hypoxic GSCs and reverse radiation resistance [97], which implied that CSC-induced chemoradiotherapy resistance should be further considered before applying redox agents. LncRNAs were also reported to participate in the process. For example, lncRNA H19 was upregulated in HCC tissues, knockdown of which caused OS and reversed the chemotherapy resistance of CD133+ CSCs via MAPK/ERK signaling [98].

Independent of CSCs, we find that the ncRNA-ROS axis can also influence chemoradiotherapy resistance through other biological processes on the basis of available evidence (Fig. 4). In breast cancer and melanoma, let-7a increased mitochondrial ROS production and improved the toxic effects of doxorubicin on cancer cells, although the underlying mechanism remained unclear [99]. MiR-17-3p elevated ROS levels by inhibiting three primary mitochondrial antioxidants, MnSOD, glutathione peroxidase 2 (Gpx2) and thioredoxin reductase 2 (TrxR2), remarkably strengthening the sensitivity of prostate cancer cells to IR [100]. MiR-34b/c prevented ubiquitin-specific protease 2a (USP2a)-c-myc-GSH signaling in prostate cancer, reversing cisplatin and doxorubicin resistance through ROS-induced apoptosis [101]. The promoters of $m i R-200 c-3 p$ and $m i R-34 a-3 p$ were occupied by zinc finger E-box binding homeobox 1 (ZEB1), which inactivated their transcription. Intriguingly, ROS-induced $m i R-200 c / 34 a$ transcription targeted ZEB1 in turn, which greatly upregulated miR-200c/34a levels, inhibiting P-glycoprotein (P-gp) expression and reversing MDR [102]. Simultaneously, ROS-induced miR-34a transcription prevented c-Met expression and enhanced the cisplatin sensitivity of HCC cells [103].

LncRNAs were also found to play a pivotal part in enhancing the sensitivity of chemoradiotherapy in multiple studies. LncRNA NLUCAT1, identified from the hypoxic status of lung adenocarcinoma cell lines,

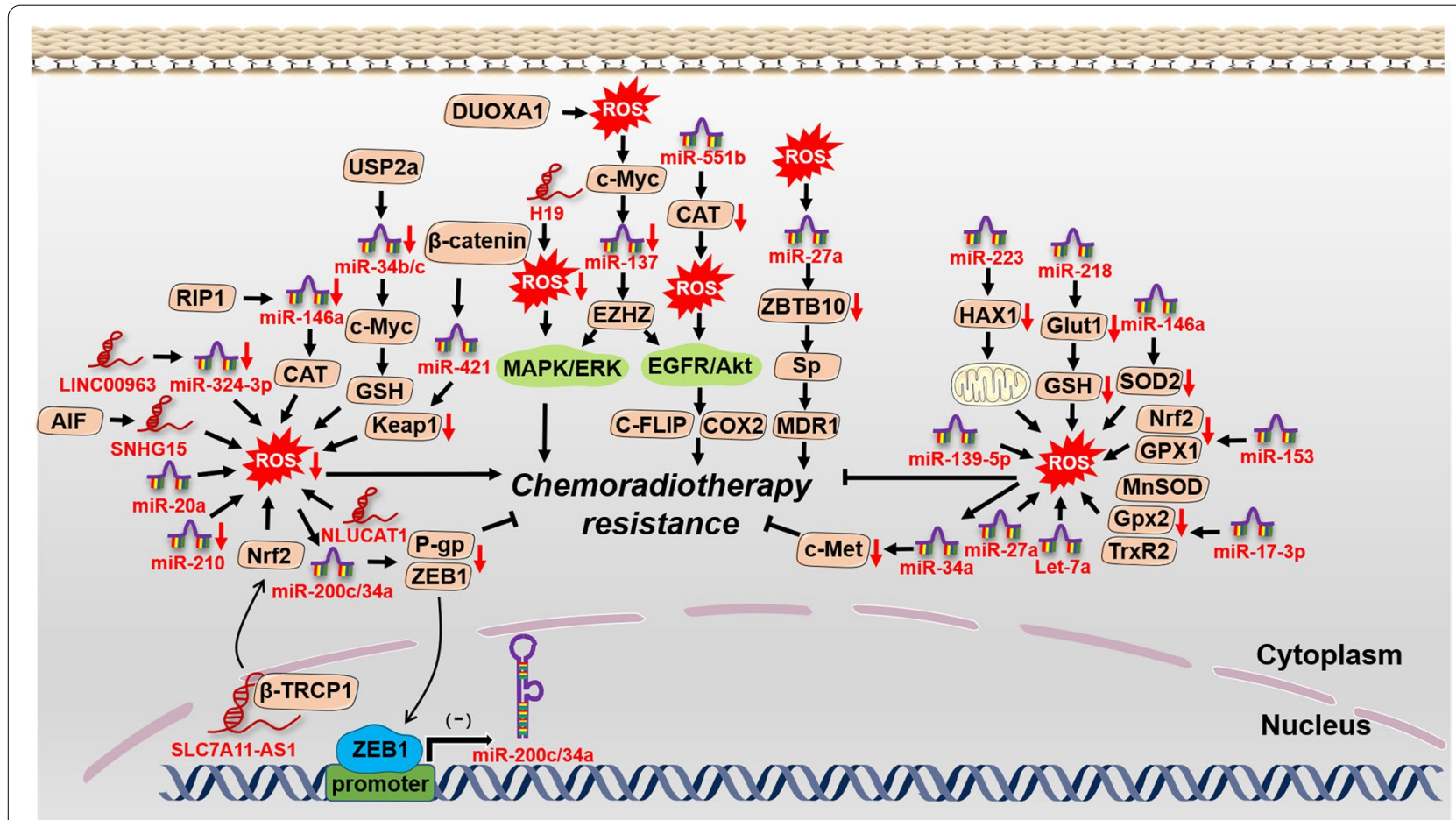

Fig. 4 The crosstalk of ROS with ncRNAs in cancer chemoradiotherapy resistance. ROS interacts with ncRNAs, influencing the effects of chemoradiotherapy. The elaborate regulatory network provides us some possible therapeutic intervention targets in chemoradiotherapy resistance 
facilitated proliferation and invasion via decreasing ROS levels with lower sensitivity of lung cancer cells to cisplatin-induced apoptosis [104]. LINC00963 was found to be elevated in several cancer types and associated with breast cancer invasion. Through sponging miR-324-3p, LINC00963 greatly relieved DNA damage and $\mathrm{OS}$, rendering cancer progression and radiotherapy resistance [105]. LncRNA SLC7A11-AS1 was identified in gemcitabine-resistant pancreatic ductal adenocarcinoma (PDAC) cells, which bound with $\beta$-TRCP1 and prevented the ubiquitination and degradation of $\mathrm{Nrf} 2$ in the nucleus. A lower level of ROS mediated by Nrf2 increased the stemness of cancer cells, which benefited drug resistance [106].

Several reports have exhibited other ncRNAs were also involved in OS-modulated chemoradiotherapy resistance. CircCCND1 was remarkably upregulated in lung cancer cells and patients, which endowed cisplatin resistance of lung cancer cells by sponging miR-187-30 to modulate OS [107]. MiR-23b-3p has been proved to be closely related to chemotherapy resistance in multiple studies [108-111]. It suggested that circZNF292, acting as a ceRNA of miR-23b-3p to regulate anti-oxidative genes, may be a potential target in reversing chemotherapy resistance [112].

\section{Therapeutic implications based on the ROS-ncRNAs axis in cancer}

Considering the great power of ROS and ncRNAs in regulating tumorigenesis and cancer progression, multiple preclinical studies targeting the ncRNAs-ROS axis have been conducted and achieved impressing treatment outcomes. ROS inducers and ncRNA-targeted or ncRNA agents are two primary druggable patterns with ncRNAs serving as both redox sensors and regulators, implying the large potential of modulating the ncRNAs-OS signaling axis in cancer therapy.

\section{ROS inducers in cancer therapy Natural/synthetic compounds}

Butyrate was found to be a ROS inducer through upregulating miR-22 expression and provoking mitochondrial dysfunction in HCC, directly causing cytochrome c release and caspase-3-dependent apoptosis [113]. Sulindac sulfide-triggered ROS decreased miR-27a expression, which prevented colon cancer cell growth via miR-27a/ ZBTB10/specificity protein (Sp) axis. A similar process also occurred in Ethyl 2-((2,3-bis (nitrooxy) propyl) disulfanyl) benzoate (GT-094)-treated colorectal cancer $[114,115]$. Phenethylisothiocyanate (PEITC) stimulated ROS generation, downregulating miR-27a/miR-20a/ miR-17 expression. The process induced miRNA-modulated ZBTB10/ZBTB4/ZBTB34 expression, inhibiting
Sp transcription factors-Sp1, Sp3, and Sp4 in pancreatic cancer cells. ROS-mediated Sp inhibition facilitated the apoptotic process, which may be a general mechanism of ROS-elevated cancer therapy [116]. However, quinacrine acted as a ROS inducer to upregulate FOXP3 by activating p38 MAPK and inactivating ERK. FOXP3-mediated miR-183 decreased $\beta$-TrCP mRNA stability, protecting Sp1 from degradation which enhanced pro-apoptotic protein Bax expression and the apoptosis of leukemia cells [117]. Besides, curcumin, betulinic acid (BA) and polygonatum odoratum lectin (POL) can also exert their antitumor effects via miRNA-mediated ROS accumulation [118-120].

\section{Nanomaterials}

With the development of nanotechnology, nano-delivery systems are promising in cancer diagnosis and therapy with lower toxicity and fewer side effects to normal cells. Among which the OS-mediated nanodrugs occupy a majority in cancer treatment. Mounting evidence has confirmed their effectiveness in preclinical studies. For example, in tumor acidy microenvironment, FeS@BSA nanocluster was degraded and produced much H2S and Fe2+, which synergistically released a ROS burst and evoked apoptosis of cancer cells [121]. Mesoporous silica nanoparticles (MSNs), featured with biochemical similarity with cell membranes, can both achieve anticancer and anti-angiogenesis purposes based on ROS [122]. Likewise, ZnO/CNT@FeO [123] and SASP/ZnO nanoparticles [124] can also increase ROS levels and facilitate cancer cell death. Though these studies are less reported about the detailed mechanisms, we hypothesis they must be closely related to the ROS-ncRNAs axis from above description.

\section{ncRNA-based agents in cancer therapy}

A plethora of annotated ROS-relevant ncRNAs have been defined as therapeutic agents or targets in cancer, primarily including miRNAs, lncRNAs and circRNAs. Among which miRNAs are the most intensively investigated due to their dual roles and simplex functional patterns in cancer biology. Thus, deleting oncogenic miRNAs with anti-miRNA oligonucleotides (AMOs) and enhancing endogenous tumor suppressor miRNAs with miRNA mimics have become two main therapeutic strategies in preclinical and clinical studies. However, lncRNAs and circRNAs act through diverse functional repertoires in cancer, targeting which will require more basic research to avoid toxic effects. Nonetheless, several reports indicate that targeting oncogenic lncRNAs/circRNAs with double-stranded RNA-mediated interference (RNAi) and single-stranded antisense oligonucleotides 
(ASOs) significantly benefits cancer therapy, suggesting ncRNAs occupy a huge potential in developing cancer drugs.

\section{MiRNAs}

MiR-1293 was identified as a candidate for miRNAbased cancer therapeutics, mimicking which would alleviate OS by targeting BRD4 and significantly prevent the growth of colorectal cells in vitro and in vivo $[125,126]$. MiR-34a was largely reported to be a tumor suppressor via silencing multiple OS-related oncogenes and signaling pathways, including SIRT1, IGF2BP3, FOXM1/eEF2K and IL-6R/STAT3 signaling axis, regulated cancer drug resistance, growth and metastasis [127-130]. Therefore, miR-34a upregulation with mimics or drugs has been widely applied in preclinical trials and shown impressive effects [131, 132]. For oncogenic miRNAs, AMO may be an option for cancer therapy. OS-mediated miR-21 was elucidated to be a pivotal oncogenic factor, that greatly facilitated cancer initiation, metastasis and drug resistance [63, 76, 97]. Yin et al exploited RNA micelles to precisely deliver the anti-miR-21 oligonucleotide into cancer cells, preventing the tumor-promoting function of miR-21 and engendering cell apoptosis in vitro and in vivo [133]. Likewise, Shu et al. developed a $15 \mathrm{~nm}$ therapeutic RNA nanoparticle to carry anti-miR-21, avoiding rapid physiological clearance and efficiently inhibiting the growth of triple negative breast cancer (TNBC) [134]. Besides miR-21, hypoxia-inducible miR-210 was also reported to prompt cancer progression in a hypoxic microenvironment $[135,136]$. Combination polymeric CXCR4 antagonist (PCX)/anti-miR-210 nanoparticles was found to elicit cancer cell death and reverse drug resistance [137].

\section{LncRNAs}

LncRNAs act as both oncogenes and tumor suppressors in cancer biology. Despite their capacity in preventing cancer progression, few preclinical studies report specific "lncRNA mimics" function in carcinogenesis. ASOs and RNAi are two major strategies to target and inhibit oncogenic lncRNAs and improve cancer therapy. LncRNA MALAT1 was found to elevate ROS levels and facilitate HNSCC metastasis [83]. Gong et al constructed MALAT1-specific ASO Au nanoparticles with nucleus-targeting TAT peptide, successfully delivering ASO into lung cancer cells and reducing metastatic tumor nodules in vivo [138]. Vascular endothelial growth factor A (VEGFA) was proved to exacerbate OS and contribute to cancer progression [139]. LINC00173.v1, another oncogenic lncRNA, was discovered to promote the angiogenesis and development of lung cancer [140]. The specific ASO against LINC00173.v1 was investigated in vivo and it revealed a better anticancer outcome and enhanced sensitivity to cisplatin in lung cancer. SiRNA-mediated RNAi has been intensively applied to silence proteincoding genes, benefiting the prognosis of multiple diseases including cancer. Silencing oncogenic lncRNAs equally improves cancer treatment. FLANC, a novel primate-specific lncRNA, was identified as a potential therapeutic target based on clinical data [141]. Encapsulating FLANC siRNA in 1,2-dioleoyl-sn-glycero-3-phosphatidylcholine nanoparticles, Pichler et al confirmed si-FLANC dramatically decreased metastases without any side effects. Mechanistically, siFLANC reduced STAT3-induced VEGFA expression, inhibiting $O S$ and cancer progression. In addition, si-MALAT1 with a nanocarrier was also an optional approach for overcoming drug resistance, which substantially enhanced the sensitivity of glioblastoma to temozolomide (TMZ) [142].

\section{CircRNAs}

CircRNAs are a novel subtype of ncRNAs identified in recent years. Nonetheless, they have been revealed to be essential in cancer biology through interacting with redox sensors or regulators, thus a therapeutic role can also be represented on the basis of circRNAs. CircMMP9 was found to act as a sponge of the redox regulator miR-124, accelerating the growth and metastasis of glioblastoma multiforme (GBM) cells $[143,144]$. The administration of circMMP9 siRNA significantly prevented cancer growth and metastasis in vitro and in vivo. Similarly, circAF4 functioned as an oncogene to sponge the other redox regulatormiR-128-3P, upregulating MLL-AF4 fusion protein expression and inducing leukemogenesis. Si-circAF4 treatment showed a remarkable cancer-preventing outcome through evoking leukemic cell apoptosis [145]. In addition to siRNAs, ASOs targeting oncogenic circRNAs also indicate the huge therapeutic potential in treating cancer. CircRNA activating MAFF (cia-MAF) was identified as a functional oncogenic circular RNA in liver cancer and liver tumorinitiating cells (TICs). The ASO specific to cia-MAF displayed impaired self-renewal and metastatic capacities of liver cancer via promoting the expression of redox sensor-MAFF [146, 147]. CircPGR was identified by circRNA sequencing (circRNA-seq) under estrogen inducement, which modulated estrogen receptor (ER)-positive breast cancer cell growth via sponging the redox regulator miR-301a-5p. CircPGR ASO administration significantly prevented the growth of ER-positive breast cancer [148, 149]. 


\section{Clinical applications of ROS-related ncRNAs in cancer patients: diagnostic value and therapeutic strategies}

In view of the potent effects of OS-related ncRNAs on all major cancer hallmarks, a multitude of preclinical research have explored the rationality of targeting these ncRNAs as mentioned above. Current evidence indicates many ROS-related ncRNAs are rather promising in cancer diagnosis and therapy, some of which have been developed as therapeutic agents and targets. Small ncRNAs such as miRNAs and siRNAs have been widely applied to clinical trials in multiple diseases, whereas fewer IncRNA or circRNA-based therapeutics have entered the clinic [150]. In this section, we summarize the clinical advancements of ROS-related ncRNAs in cancer diagnosis and therapy, emphasizing more efforts should be made to test and develop more ncRNA-based drugs. Partial ROS-related ncRNAs that enter the clinic are listed in Table 1.

\section{Cancer diagnosis MiRNAs}

MiR-373 functioned as a redox regulator and was found to promote tumorigenesis in multiple cancers [72, 151, 152]. In a clinical study concerning the diagnosis of breast cancer, miR-373 was exploited as a serum biomarker to evaluate its correlation with clinicopathological documented data, staging, grading and tumor receptors (NCT04720508). Similarly, some research reported that miR-155 was oncogenic in several cancers through targeting Nrf2-mediated OS [153-155], which was considered as a promising biomarker coupled with telomerase reverse transcriptase (TERT) in a clinical exploration through assessing the potential roles in the diagnosis of non-muscle-invasive bladder cancer and their correlation with stage and grade (NCT03591367). Another oncogenic redox regulator miR-371, was largely reported to cause the growth, metastasis and drug resistance of cancers [72, 156-158]. On the foundation of differentially expressed levels, Nichols et al carried out a clinical trial to estimate the positive predictive value of miR-371 in patients with recurrent germ cell cancers (NCT04435756). In pancreatic cancer, limited by the specificity of the FDA-approved biomarker CA199, small RNAs have been considered as promising biomarkers owing to their numbers and stability, miRNAs included. These small RNAs from circulating extracellular exosomes between pancreatic cancer patients and healthy controls are being analyzed to discover specific diagnostic biomarkers through next-generation sequencing (NCT04636788). Similarly, another clinical trial exploited urinary exosomes to discern and validate the predictive role of candidate exosomal microRNAs in aggressive prostate cancer (NCT03911999). Nonspecific to a certain miRNA (34 miRNAs were assessed), a study attempted to search for specific miRNA markers for earlier diagnosis of lung cancer by quantifying miRNAs from peripheral blood (NCT03293433).

\section{LnCRNAs and circRNAs}

LncRNA and circRNA-based clinical research are less conducted except for the redox regulator-lncRNA HOTAIR (NCT03469544). Nonetheless, the whole lncRNA/circRNA sequencing to uncover novel biomarkers has been carried out. For instance, in neuroendocrine neoplasm (NEN), circular RNA sequencing was used to screen candidate circRNAs that potentially acted as diagnostic and predictive biomarkers, following subsequent validation between the neuroendocrine tumor group and control group (NCT04464122). Likewise, IncRNA

Table 1 Clinical applications of partial ROS-related ncRNAs in cancer patients

\begin{tabular}{|c|c|c|c|c|c|}
\hline NcRNA & Type & Cancer & Application & Status & Identifier \\
\hline MiR-373 & NA & Breast cancer & Cancer diagnosis & Recruiting & NCT04720508 \\
\hline MiR-155 & NA & Bladder cancer & Cancer diagnosis & Completed & NCT03591367 \\
\hline MiR-371 & NA & Germ cell cancer & Cancer diagnosis & Recruiting & NCT04435756 \\
\hline HOTAIR & NA & Thyroid cancer & Cancer diagnosis & Unknown & NCT03469544 \\
\hline MiR-16 & Mimic & $\begin{array}{l}\text { Pleural mesothelioma lung } \\
\text { cancer }\end{array}$ & Cancer therapy & Phase I & NCT02369198 \\
\hline MiR-122 & ASO & Liver cancer & Cancer therapy & Phase I/II & $\begin{array}{l}\text { NCT01646489, NCT01727934, } \\
\text { NCT01872936, NCT01200420 }\end{array}$ \\
\hline MiR-155 & ASO & T-cell lymphoma & Cancer therapy & Phase II & NCT03713320, NCT03713320 \\
\hline MiR-34a & Mimic & Melanoma & Cancer therapy & Phase I & $\begin{array}{l}\text { NCT01829971, } \\
\text { NCT02862145 }\end{array}$ \\
\hline MtIncRNA & ASO & Unresectable tumors & Cancer therapy & Phase I & $\begin{array}{l}\text { NCT02508441, } \\
\text { NCT03985072 }\end{array}$ \\
\hline
\end{tabular}


sequencing can also benefit cancer diagnosis. Considering the availability and detectability of exosomes, two clinical trials tried to extract exosomal RNAs from the plasma of patients with lung cancer/high grade serous ovarian cancer (HGSOC) and healthy individuals, aiming to identify multiple specific lncRNA and lncRNA/ miRNA biomarkers through next-generation RNA sequencing (NCT03830619, NCT03738319). In addition, RNA profiling through the whole RNA sequencing of clinical specimens may suggest some unexpected lncRNA/circRNA biomarkers in cancer diagnosis. In metastatic high-grade osteosarcoma, Shen et al collected blood samples between metastatic and non-metastatic osteosarcoma patients to extract exosomes and perform RNA profiling, aiming to identify specific RNA biomarkers for osteosarcoma metastasis through next-generation RNA sequencing (NCT03108677). We believe these explorations will conduce to observing more valuable OS-related lncRNA/circRNA biomarkers based on clinical specimens.

ROS-related ncRNAs not only serve as direct biomarkers to help distinguish cancer patients, but also facilitate clinicians to monitor redox homeostasis and evaluate the risk of suffering redox-related diseases. Thus, identifying more ROS-related ncRNA biomarkers is essential for diagnosing redox-related diseases including cancer at an early stage.

\section{Cancer therapy MiRNAs}

Though miRNA-mediated preclinical studies with significant anticancer effects have been extensively reported, relevant clinical trials are less carried out. MiR-16 was able to downregulate OS and inhibit the progression of multiple cancers, functioning as a tumor suppressor in malignant pleural mesothelioma, lung cancer breast cancer and leukemia [159-163]. In malignant pleural mesothelioma and non-small cell lung cancer, miR-16 mimic was packaged in minicells targeted with epidermal growth factor receptor (EGFR)-specific antibodies, which exhibited an acceptable safety profile in a phase I study [164] (NCT02369198). Some miRNA-based drugs may not be tested directly for cancers in clinical trials, but we speculate they do good to cancer prevention as well. For example, the hepatitis $\mathrm{C}$ virus (HCV) infection has been proved to be closely related to HCC, avoiding which is essential to HCC prevention. Miravirsen (SPC3649), a developed anti-miR-122 agent, was considered to efficiently alleviate $\mathrm{HCV}$ infection in multiple phase I/II investigations (NCT01646489, NCT01727934, NCT01872936, NCT01200420). However, some other miRNA-based clinical trials were terminated due to the limited safety or efficacy. Several findings uncovered
miR-155 was capable of inducing OS and engendering cancer metastasis and chemoresistance via multiple genes and signaling pathways [165-167], targeting and inhibiting which may benefit cancer treatment. Two phase II investigations were designed to evaluate the efficacy and safety of cobomarsen (a miR-155 inhibitor) in patients with cutaneous T-cell lymphoma (CTCL) and mycosis fungoides (MF), accompanied by a comparation to vorinostat, a drug that has been approved for the treatment of CTCL (NCT03713320, NCT03713320). In addition, miR-34a was universally identified as a tumor suppressor through redox regulation, whose analogues were widely applied in animal models [78, 101, 102]. MRX34, a miR34a mimic, was exploited as an intervention agent to test the safety, pharmacokinetics and pharmacodynamics in a phase I study (NCT01829971) and assess the efficacy of combined treatment with dexamethasone, a phase II drug for melanoma patients (NCT02862145). Unfortunately, these promising clinical studies were forced to end on account of the significant side effects and insufficient efficiency.

\section{LncRNAs}

Most investigations targeting conventional lncRNAs are still in the preclinical stage, though a multitude of inspiring results have suggested that IncRNA-based drugs are very promising. Even so, it is conceivable that the entrance of IncRNA-based therapeutics into clinical studies is imminent. Herein, we notice that mitochondrial lncRNAs (mtlncRNA), specifically transcribed by mitochondrial DNA, have obtained rapid advancements [168]. Antisense noncoding mitochondrial RNA (ASncmtRNA) was identified as a redox sensor, with an oncogenic role in the progression of various cancers [169-171]. These preliminary results implied the potential of ASncmtRNA-targeting ASOs in clinical cancer treatment. To this end, Andes-1537 was approved to treat multiple advanced unresectable solid tumors by targeting ASncmtRNA with a short single-stranded phosphorothioate ASO (NCT02508441, NCT03985072). The phase I study demonstrated that Andes-1537 is a well-tolerated drug [172].

\section{Natural compounds targeting ROS-related ncRNAs}

Additionally, though not claiming lncRNAs or circRNAs as direct targets, some natural compounds have entered the clinic and been exploited as anticancer agents. Curcumin was found to relieve OS through modulating Nrf2 expression, exerting antitumor activity and enhancing the sensitivity of chemoradiotherapy via lncRNA and circRNA regulation [173-178]. A clinical practice used for observing the roles of curcumin in intestinal adenomas showed neither significant adverse effects nor anticancer 
functions (NCT00927485). Dietary supplementation with curcumin to assess the radiosensitizing and radioprotective effects in prostate cancer was completed in a clinical trial, though the detailed results were not posted (NCT01917890). Another phase II clinical study tested the function and safety of curcumin in advanced pancreatic cancer, suggesting the huge potential of curcumin in clinical application [179] (NCT00094445). Similarly, resveratrol acted as a redox regulator, remarkably decreasing OS levels and inhibiting cancer development through activating sirtuin 1 (SIRT1) and lncRNA-modulated pathways [180, 181]. Clinical trials have been exploited to investigate the therapeutic outcomes of resveratrol in colorectal cancer (NCT00256334, NCT02261844) and liver cancer (NCT02261844), which exhibited an impressing tumor preventive role in cancer patients [182].

\section{Concluding remarks and future perspectives}

OS is a double-edged sword. Oxidative eustress activates redox signaling and promotes growth and migration. Nevertheless, oxidative distress stimulates oxidative damage, growth inhibition and cell death. The dual roles remind us of two types of therapeutic methods targeting ROS. Though some antioxidant agents have been elucidated to efficiently prevent oxidative damage and cancer development, they may not always benefit or even be at risk. Most treatments are still to increase ROS levels that partially owe to breaking the redox equilibrium of cancer cells. Although the strategies have acquired gratifying achievements, disadvantages such as low sensitivity of cancer cells, low abundance within cancer cells and side effects on normal cells are equally significant. Therefore, ROSbased treatments should be revisited from a whole new perspective.

NcRNAs, especially for miRNAs and lncRNAs, can interact with ROS in cancer development and chemotherapy/radiotherapy resistance. Given the strong tissue and space-time specificity and conservative property of ncRNAs, directly developing ROS-based ncRNA and ncRNA-targeted drugs may cure the specific cancer types. In addition, the functional mechanisms of ncRNAs are simplex, making it probable that exploit highly targeted and low-toxicity ncRNA agents. With the rapid development of biotechnologies, more ncRNAs are being discovered, and their precise regulatory patterns will be identified. It is capable to establish a biobank, aiming

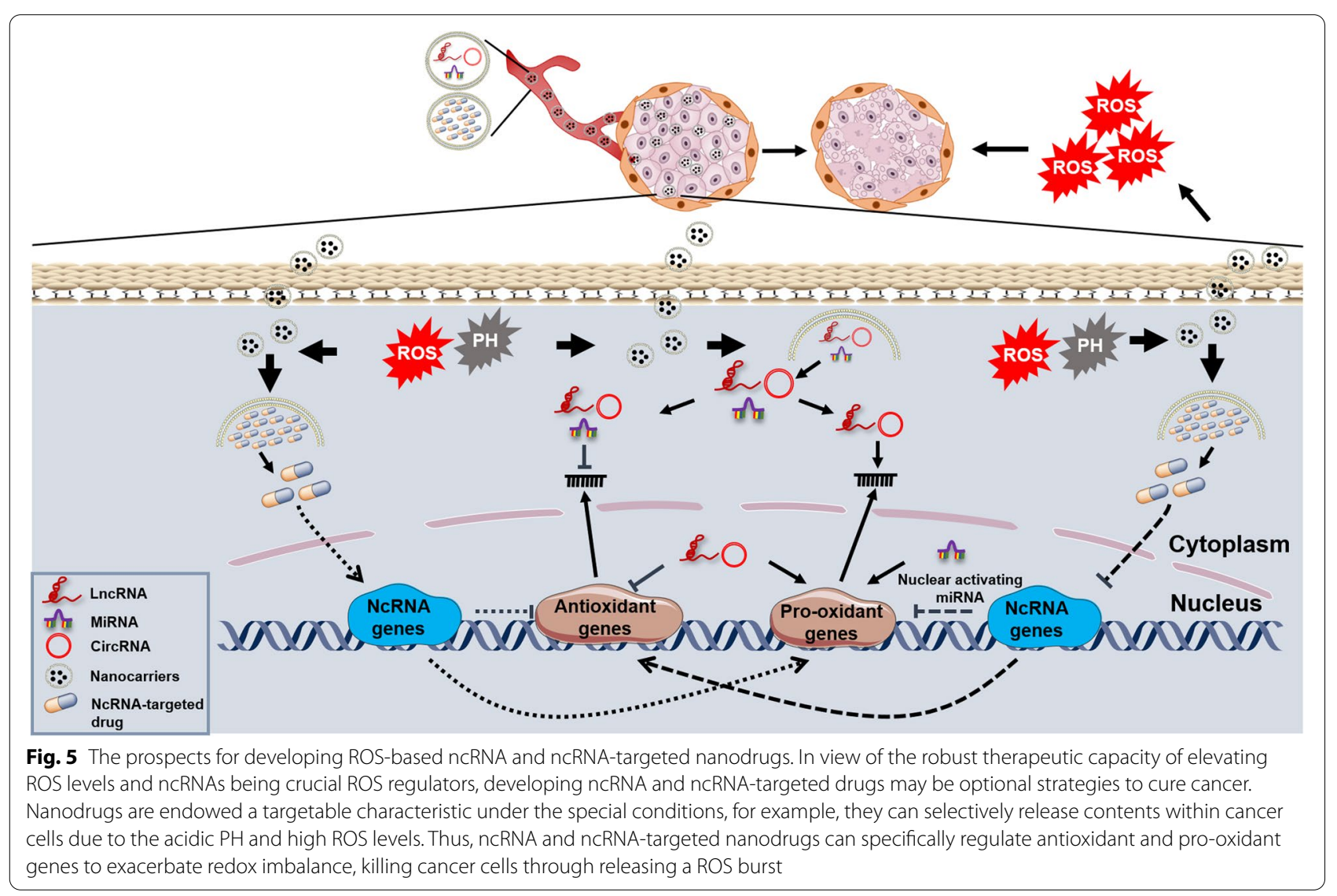


to illuminate the ncRNA-ROS interaction network and promote the progression of ROS-based ncRNA or ncRNA-targeted medicine. Additionally, more advanced nanocarriers will make it promising that all different kinds of ncRNAs can be packaged and quickly enter cancer cells regardless of their length and biocompatibility (Fig. 5).

Notably, the tissue specificity of ncRNAs suggests that they may occupy significant parts in tissue formation, growth, differentiation, development, etc. The altered expression of ncRNAs with human intervention may be a risk on account of upregulating or downregulating ncRNAs can both cause tissue dysfunction and even damage. Aerobic glycolysis, which frequently occurs in multiple tumor tissues and determines the survival of cancer cells, is a crucial target for the ROS-ncRNA axis in cancer therapy. However, we can never only focus on the process to treat cancers. Mounting evidence indicates that in different cancer types and progressive stages, cancer cells exhibit unique metabolic forms. Furthermore, there are still many other challenges limiting the imagination. Most lncRNAs remain unexplored, which is a large obstacle for establishing a complete ncRNA-ROS interaction network to find more therapeutics. These are considerable for safe precision medicine, thereby the extensive basic research and long-term clinical observations must be carried out before relevant drugs being developed and entering the markets. Altogether, ROS-based ncRNA or ncRNA-targeted medicine holds promise in cancer therapy but still requires more investigations to ensure their safety and effectiveness.

\section{Abbreviations}

OS: Oxidative stress; ROS: Reactive oxygen species; NcRNAs: Noncoding RNAs; RNS: Reactive nitrogen species; RSS: Reactive sulfur species; RCS: Reactive chlorine species; ER: Endoplasmic reticulum; MiRNAs: MicroRNAs; CircRNAs: Circular RNAs; SiRNAs: Small interfering RNAs; SnRNAs: Small nuclear RNAs; SnoRNAs: Small nucleolar RNAs; PiRNAs: PIWI-interacting RNAs; tsRNAs: tRNAderived small RNAs; eRNAs: enhancer noncoding RNAs; SOD: Superoxide dismutase; CAT: Catalase; GPX: Glutathione peroxidase; NADPH: Nicotinamide adenine dinucleotide phosphate; GSH: Glutathione; Nrf2: Nuclear factor erythroid 2-related factor 2; CSCs: Cancer stem cells; PDCD4: Programmed cell death 4; IR: Ionizing radiation; HIF-1a: Hypoxia-inducible factor-1 a; COX-2: Cyclooxygenases-2; HCC: Hepatocellular carcinoma; GC: Gastric cancer; GAS5: LncRNA growth arrest-specific transcript 5; EMT: Epithelial-mesenchymal transition; EMT-TFs: EMT-activating transcription factors; MMP-9: metalloproteases-9; TXNIP: Thioredoxin-interacting protein; MTP18: Mitochondrial protein 18 kDa; NRP1: Neuropilin-1; TGFBR2: TGF- $\beta$ receptor 2; HNSCC: Head and neck squamous cell carcinoma; MDR: Multidrug resistance; TNBCSCs: Triplenegative breast cancer stem cells; GSCs: Glioma stem cells; Gpx2: Glutathione peroxidase 2; TrxR2: Thioredoxin reductase 2; USP2a: Ubiquitin-specific protease 2a; ZEB1: Zinc finger E-box binding homeobox 1; P-gp: P-glycoprotein; PDAC: Pancreatic ductal adenocarcinoma; Sp: Specificity protein; PEITC: Phenethylisothiocyanate; BA: Betulinic acid; POL: Polygonatum odoratum lectin; MSNs: Mesoporous silica nanoparticles; AMOs: Anti-miRNA oligonucleotides; RNAi: RNA-mediated interference; ASOs: Antisense oligonucleotides; TNBC: Triple negative breast cancer; PCX: polymeric CXCR4 antagonist; VEGFA: Vascular endothelial growth factor A;TMZ:Temozolomide; GBM: Glioblastoma multiforme; Cia-MAF: CircRNA activating MAFF; TICs: Tumor-initiating cells;
CircRNA-seq: CircRNA sequencing; ER: Estrogen receptor; TERT: Telomerase reverse transcriptase; NEN: Neuroendocrine neoplasm; HGSOC: High grade serous ovarian cancer; EGFR: Epidermal growth factor receptor; HCV: Hepatitis C virus; CTCL: Cutaneous T-cell lymphoma; MF: Mycosis fungoides; MtIncRNA: Mitochondrial IncRNAs; ASncmtRNA: Antisense noncoding mitochondrial RNA; SIRT1: Sirtuin 1

\section{Acknowledgements}

Not applicable.

\section{Authors' contributions}

STZ, CHH and PW conceived the structure of the manuscript. JZ, ZZ, MML and YY drafted initial manuscript. STZ, CHH and PW revised the manuscript. JZ and $\mathrm{BHZ}$ prepared the figures. All authors read and approved the final manuscript.

\section{Funding}

This work was supported by grants from the National Key Research and Development Project (2020YFA0509400, 2021YFC2009100, 2021YFC2009102, 2017YFA0106800 and 2018YFA0109200), Guangdong Basic and Applied Basic Research Foundation (2019B030302012), the National Natural Science Foundation of China (81821002, 81790251, 82130082 and 81822034), the Key Research Programs of Science and Technology Department of Sichuan Province (2021YFS0015), Sichuan Science-Technology International Cooperation Project (2019YFH0144) and Direct Scientific Research Grants from West China Second Hospital, Sichuan University (KS021 and K1907).

Availability of data and materials

Not applicable.

\section{Declarations}

Ethics approval and consent to participate

Not applicable.

\section{Consent for publication}

All authors consent to publication.

\section{Competing interests}

The authors declare that they have no competing interests.

Received: 10 November 2021 Accepted: 26 December 2021

Published online: 26 January 2022

\section{References}

1. Kano G, et al. Mechanism of Siglec-8-mediated cell death in IL-5-activated eosinophils: role for reactive oxygen species-enhanced MEK/ERK activation. J Allergy Clin Immunol. 2013;132(2):437-45.

2. Zhang T, et al. Cucurbitacin induces autophagy through mitochondrial ROS production which counteracts to limit caspase-dependent apoptosis. Autophagy. 2012;8(4):559-76.

3. Liu Y, et al. Nuclear lactate dehydrogenase a senses ROS to produce a-hydroxybutyrate for HPV-induced cervical tumor growth. Nat Commun. 2018;9(1):4429.

4. Zhu M, Dai X. Maintenance of translational elongation rate underlies the survival of Escherichia coli during oxidative stress. Nucleic Acids Res. 2019;47(14):7592-604

5. Zhou Z, et al. Erythrocytes from patients with type 2 diabetes induce endothelial dysfunction via Arginase I. J Am Coll Cardiol. 2018;72(7):769-80.

6. Patel $P$, et al. Damage sensing by a Nox-Ask1-MKK3-p38 signaling pathway mediates regeneration in the adult drosophila midgut. Nat Commun. 2019;10(1):4365.

7. Zhang $X$, et al. Redox signals at the ER-mitochondria interface control melanoma progression. EMBO J. 2019;38(15):e100871.

8. Makhezer N, et al. NOX1-derived ROS drive the expression of Lipocalin-2 in colonic epithelial cells in inflammatory conditions. Mucosal Immunol. 2019;12(1):117-31. 
9. Xie X, et al. Autophagy is induced through the ROS-TP53-DRAM1 pathway in response to mitochondrial protein synthesis inhibition. Autophagy. 2012;8(7):1071-84.

10. Hamarsheh $\mathrm{S}$, et al. Oncogenic Kras causes myeloproliferation via NLRP3 inflammasome activation. Nat Commun. 2020;11(1):1659.

11. Zhang $P$, et al. Oxidative stress and diabetes: antioxidative strategies. Front Med. 2020;14(5):583-600.

12. Zhang Y, et al. NADPH oxidases and oxidase crosstalk in cardiovascular diseases: novel therapeutic targets. Nat Rev Cardiol. 2020;17(3):170-94.

13. Huang $Y$, et al. Identification of a fluorescent small-molecule enhancer for therapeutic autophagy in colorectal cancer by targeting mitochondrial protein translocase TIM44. Gut. 2018;67(2):307-19.

14. Simic MG, Bergtold DS, Karam LR. Generation of oxy radicals in biosystems. Mutation Res/Fundamental Mol Mechan Mutagene. 1989;214(1):3-12.

15. Gorrini C, Harris IS, Mak TW. Modulation of oxidative stress as an anticancer strategy. Nat Rev Drug Discov. 2013;12(12):931-47.

16. Pratheeshkumar $\mathrm{P}$, et al. Oncogenic transformation of human lung bronchial epithelial cells induced by arsenic involves ROSdependent activation of STAT3-miR-21-PDCD4 mechanism. Sci Rep. 2016;6:37227.

17. Zhang $X$, et al. MicroRNA-21 modulates the levels of reactive oxygen species by targeting SOD3 and TNFa. Cancer Res. 2012;72(18):4707-13.

18. Hou L, et al. Induction of miR-21-PDCD4 signaling by UVB in JB6 cells involves ROS-mediated MAPK pathways. Exper Toxicol Pathol. 2013:65(7-8):1145-8.

19. Tan J, et al. An R-loop-initiated CSB-RAD52-POLD3 pathway suppresses ROS-induced telomeric DNA breaks. Nucleic Acids Res. 2020;48(3):1285-300.

20. Khan S, Zafar A, Naseem I. Redox cycling of copper by coumarin-di(2picolyl) amine hybrid molecule leads to ROS-mediated modulation of redox scavengers, DNA damage and cell death in diethylnitrosamine induced hepatocellular carcinoma. Bioorg Chem. 2020;99:103818.

21. Liu N, et al. Vitexin compound 1, a novel extraction from a Chinese herb, suppresses melanoma cell growth through DNA damage by increasing ROS levels. J Exper Clin Cancer Res. 2018;37(1):269.

22. Fimognari $\mathrm{C}$. Role of oxidative RNA damage in chronic-degenerative diseases. Oxidative Med Cell Longev. 2015;2015:358713.

23. Willi J, et al. Oxidative stress damages rRNA inside the ribosome and differentially affects the catalytic center. Nucleic Acids Res. 2018;46(4):1945-57.

24. Veskoukis AS, Tsatsakis AM, Kouretas D. Dietary oxidative stress and antioxidant defense with an emphasis on plant extract administration. Cell Stress Chaperones. 2012;17(1):11-21.

25. Stepniak J, Karbownik-Lewinska M. 17ß-estradiol prevents experimentally-induced oxidative damage to membrane lipids and nuclear DNA in porcine ovary. Syst Biol Reprod Med. 2016;62(1):17-21.

26. Félix $R$, et al. Evaluating the in vitro potential of natural extracts to protect lipids from oxidative damage. Antioxidants (Basel, Switzerland). 2020;9(3):231

27. Li X, et al. Renal purge of Hemolymphatic lipids prevents the accumulation of ROS-induced inflammatory oxidized lipids and protects drosophila from tissue damage. Immunity. 2020;52(2):374-387.e6.

28. Place RF, et al. MicroRNA-373 induces expression of genes with complementary promoter sequences. Proc Natl Acad Sci U S A. 2008;105(5):1608-13.

29. Xiao M, et al. MicroRNAs activate gene transcription epigenetically as an enhancer trigger. RNA Biol. 2017;14(10):1326-34

30. Jian X, et al. Hsa_circ_001680 affects the proliferation and migration of CRC and mediates its chemoresistance by regulating BMl1 through miR-340. Mol Cancer. 2020;19(1):20.

31. Wang W, et al. Long non-coding RNA UCA1 promotes malignant phenotypes of renal cancer cells by modulating the miR-182-5p/DLL4 axis as a ceRNA. Mol Cancer. 2020;19(1):18.

32. Fabbri $M$, et al. Decrypting noncoding RNA interactions, structures, and functional networks. Genome Res. 2019;29(9):1377-88.

33. Zhang $X$, et al. Role of non-coding RNAs and RNA modifiers in cancer therapy resistance. Mol Cancer. 2020;19(1):47.

34. Kuhlmann J, et al. Circulating U2 small nuclear RNA fragments as a novel diagnostic tool for patients with epithelial ovarian cancer. Clin Chem. 2014;60(1):206-13.
35. Baraniskin A, et al. Circulating U2 small nuclear RNA fragments as a novel diagnostic biomarker for primary central nervous system lymphoma. Neuro-oncology. 2016;18(3):361-7.

36. Sousa A, et al. Nanotechnology-based siRNA delivery strategies for metastatic colorectal cancer therapy. Int J Pharm. 2019;568:118530.

37. Aghamiri S, et al. Nanoparticle-siRNA: a potential strategy for ovarian cancer therapy? Nanomedicine (London, England). 2019;14(15):2083-100.

38. Deng W, et al. Hsa_circRNA 101036 acts as tumor-suppressor in oral squamous cell carcinoma cells via inducing endoplasmic reticulum stress. Eur Rev Med Pharmacol Sci. 2020;24(11):6111-21.

39. Zorov DB, Juhaszova M, Sollott SJ. Mitochondrial reactive oxygen species (ROS) and ROS-induced ROS release. Physiol Rev. 2014;94(3):909-50.

40. Zhao Y, et al. ROS signaling under metabolic stress: cross-talk between AMPK and AKT pathway. Mol Cancer. 2017;16(1):79.

41. DeNicola GM, et al. Oncogene-induced Nrf2 transcription promotes ROS detoxification and tumorigenesis. Nature. 2011;475(7354):106-9.

42. Jin F, et al. The PI3K/Akt/GSK-3ß/ROS/elF2B pathway promotes breast cancer growth and metastasis via suppression of NK cell cytotoxicity and tumor cell susceptibility. Cancer Biol Med. 2019;16(1):38-54.

43. Chen C, Wang S, Liu P. Deferoxamine enhanced mitochondrial iron accumulation and promoted cell migration in triple-negative MDAMB-231 breast cancer cells via a ROS-dependent mechanism. Int J Mol Sci. 2019;20(19):4952.

44. Liu J, et al. Topoisomerase inhibitors promote cancer cell motility via ROS-mediated activation of JAK2-STAT1-CXCL1 pathway. J Exper Clin Cancer Res. 2019;38(1):370.

45. Wang $C$, et al. Targeting IRS-1/mPGES-1/NOX2 to inhibit the inflammatory response caused by insulin-likegrowth factor---induced activation of NF-KB and NLRP3 in cancer cells. Vet Comp Oncol. 2020;18(4):689-98.

46. Deng W, et al. MICAL1 facilitates breast cancer cell proliferation via ROSsensitive ERK/cyclin D pathway. J Cell Mol Med. 2018;22(6):3108-18.

47. Locasale JW, Cantley LC. Metabolic flux and the regulation of mammalian cell growth. Cell Metab. 2011;14(4):443-51.

48. Sosa V, et al. Oxidative stress and cancer: an overview. Ageing Res Rev. 2013;12(1):376-90.

49. Mosińska P, et al. Dual functional capability of dendritic cells - cytokineinduced killer cells in improving side effects of colorectal cancer therapy. Front Pharmacol. 2017;8:126.

50. Schmidlin C, et al. The intricacies of NRF2 regulation in cancer. Semin Cancer Biol. 2021;76:110-9.

51. Liao Z, Chua D, Tan NS. Reactive oxygen species: a volatile driver of field cancerization and metastasis. Mol Cancer. 2019;18(1):65.

52. Hayes JD, Dinkova-Kostova AT, Tew KD. Oxidative stress in cancer. Cancer Cell. 2020;38:167-97.

53. Tang J, et al. Oxidative stress-modulating drugs have preferential anticancer effects - involving the regulation of apoptosis, DNA damage, endoplasmic reticulum stress, autophagy, metabolism, and migration. Semin Cancer Biol. 2019;58:109-17.

54. Singhal R, et al. HIF-2a activation potentiates oxidative cell death in colorectal cancers by increasing cellular iron. J Clin Invest. 2021;131(12):e143691.

55. Weng W, et al. Novel evidence for a PIWI-interacting RNA (piRNA) as an oncogenic mediator of disease progression, and a potential prognostic biomarker in colorectal cancer. Mol Cancer. 2018;17(1):16.

56. Qian X, et al. LCAT3, a novel m6A-regulated long non-coding RNA, plays an oncogenic role in lung cancer via binding with FUBP1 to activate c-MYC. J Hematol Oncol. 2021;14(1):112.

57. Huang X, et al. LINC00842 inactivates transcription co-regulator PGC-1a to promote pancreatic cancer malignancy through metabolic remodelling. Nat Commun. 2021;12(1):3830.

58. Zhang $\mathrm{Q}$, et al. Pulmonary aerosol delivery of let-7b microRNA confers a striking inhibitory effect on lung carcinogenesis through targeting the tumor immune microenvironment. Adv Sci. 2021;8(17):e2100629.

59. Wang $\mathrm{H}$, et al. IncRNA SNHG6 promotes hepatocellular carcinoma progression by interacting with HNRNPL/PTBP1 to facilitate SETD7/LZTFL1 mRNA destabilization. Cancer Lett. 2021;520:121-31.

60. Zhu G, et al. HOXBLINC long non-coding RNA activation promotes leukemogenesis in NPM1-mutant acute myeloid leukemia. Nat Commun. 2021;12(1):1956. 
61. Dakup P, Gaddameedhi S. Impact of the circadian clock on UV-induced DNA damage response and Photocarcinogenesis. Photochem Photobiol. 2017;93(1):296-303.

62. Leng S, et al. Radon exposure, IL-6 promoter variants, and lung squamous cell carcinoma in former uranium miners. Environ Health Perspect. 2016;124(4):445-51.

63. PratheeshkumarP,etal. Hexavalentchromiuminduces malignant transformation of human lung bronchial epithelial cells via ROS-dependent activation of miR-21-PDCD4 signaling. Oncotarget. 2016;7(32):51193-210.

64. He J, et al. Chronic arsenic exposure and angiogenesis in human bronchial epithelial cells via the ROS/miR-199a-5p/HIF-1a/COX-2 pathway. Environ Health Perspect. 2014;122(3):255-61.

65. Xiang S, et al. LnCRNA IDH1-AS1 links the functions of C-Myc and HIF1a via IDH1 to regulate the Warburg effect. Proc Natl Acad Sci U S A. 2018;115(7):E1465-74.

66. Zuo K, et al. The expression and role of IncRNA AX800134 in hepatitis B virus-related hepatocellular carcinoma. Virus Genes. 2018;54(4):475-83.

67. Zhao Y, et al. The IncRNA MACC1-AS1 promotes gastric cancer cell metabolic plasticity via AMPK/Lin28 mediated mRNA stability of MACC1. Mol Cancer. 2018;17(1):69.

68. Xu W, et al. Long non-coding RNA GAS5 accelerates oxidative stress in melanoma cells by rescuing EZH2-mediated CDKN1C downregulation. Cancer Cell Int. 2020;20(1):116.

69. Han D, et al. The tumor-suppressive human circular RNA CirclTCH sponges miR-330-5p to ameliorate doxorubicin-induced Cardiotoxicity through Upregulating SIRT6, Survivin, and SERCA2a. Circ Res. 2020;127(4):e108-25.

70. Feng D, et al. Circ-PRKCB acts as a ceRNA to regulate p66Shc-mediated oxidative stress in intestinal ischemia/reperfusion. Theranostics. 2020;10(23):10680-96.

71. Brabletz T, et al. EMT in cancer. Nat Rev Cancer. 2018;18(2):128.

72. Chen D, et al. MiR-373 drives the epithelial-to-mesenchymal transition and metastasis via the miR-373-TXNIP-HIF1a-TWIST signaling axis in breast cancer. Oncotarget. 2015;6(32):32701-12.

73. Zhang Y, et al. MTP18 overexpression contributes to tumor growth and metastasis and associates with poor survival in hepatocellular carcinoma. Cell Death Dis. 2018;9(10):956.

74. Singh A, et al. Transcription factor NRF2 regulates miR-1 and miR-206 to drive tumorigenesis. J Clin Invest. 2013;123(7):2921-34.

75. Yin K, et al. MiR-206 suppresses epithelial mesenchymal transition by targeting TGF- $\beta$ signaling in estrogen receptor positive breast cancer cells. Oncotarget. 2016;7(17):24537-48.

76. Guo Y, et al. Kallistatin inhibits TGF- $\beta$-induced endothelial-mesenchymal transition by differential regulation of microRNA-21 and eNOS expression. Exp Cell Res. 2015;337(1):103-10.

77. Li GY, et al. Long non-coding RNAs AC026904.1 and UCA1: a "one-two punch" for TGF- $\beta$-induced SNAI2 activation and epithelial-mesenchymal transition in breast cancer. Theranostics. 2018;8(10):2846-61.

78. Yan X, et al. Mesenchymal stem cells promote Hepatocarcinogenesis via IncRNA-MUF interaction with ANXA2 and miR-34a. Cancer Res. 2017;77(23):6704-16.

79. Wang $H$, et al. Lnc-SNHG1 activates the TGFBR2/SMAD3 and RAB11A/ Wnt/ $\beta$-catenin pathway by sponging MiR-302/372/373/520 in invasive pituitary tumors. Cell Physiol Biochem. 2018;48(3):1291-303.

80. Yeh L-Y, et al. miR-372 inhibits p62 in head and neck squamous cell carcinoma in vitro and in vivo. Oncotarget. 2015;6(8):6062-75.

81. Li S-Z, et al. MicroRNA-34a induces apoptosis in the human glioma cell line, $A 172$, through enhanced ROS production and NOX2 expression. Biochem Biophys Res Commun. 2014;444(1):6-12.

82. Amodio N, et al. Drugging the InCRNA MALAT1 via LNA gapmeR ASO inhibits gene expression of proteasome subunits and triggers antimultiple myeloma activity. Leukemia. 2018;32(9):1948-57.

83. Wang $Y$, et al. TGF- $\beta$-induced STAT3 overexpression promotes human head and neck squamous cell carcinoma invasion and metastasis through malat1/miR-30a interactions. Cancer Lett. 2018;436:52-62.

84. Deng $X$, et al. PM2.5 exposure-induced autophagy is mediated by IncRNA loc146880 which also promotes the migration and invasion of lung cancer cells. Biochim Biophys Acta, Gen Subj. 2017;1861(2):112-25.

85. Yin D, et al. Ropivacaine inhibits cell proliferation, migration and invasion, whereas induces oxidative stress and cell apoptosis by circSCAF11/ miR-145-5p Axis in Glioma. Cancer Manag Res. 2020;12:11145-55.
86. Jung $Y$, et al. Epigenetic regulation of miR-29a/miR-30c/DNMT3A axis controls SOD2 and mitochondrial oxidative stress in human mesenchymal stem cells. Redox Biol. 2020;37:101716.

87. Wang $L$, et al. MiR-30c-5p mediates the effects of panax notoginseng saponins in myocardial ischemia reperfusion injury by inhibiting oxidative stress-induced cell damage. Biomed Pharmacother= Biomedecine \& pharmacotherapie. 2020;125:109963.

88. Sun M, et al. MicroRNA-30c-5p protects against myocardial ischemia/ reperfusion injury via regulation of Bach1/Nrf2. Toxicol Appl Pharmacol. 2021;426:115637.

89. Guo Y, et al. Circ3823 contributes to growth, metastasis and angiogenesis of colorectal cancer: involvement of miR-30c-5p/TCF7 axis. Mol Cancer. 2021;20(1):93.

90. Hu Z, et al. Circular RNA sequencing identifies CircASAP1 as a key regulator in hepatocellular carcinoma metastasis. Hepatology (Baltimore, Md). 2020;72(3):906-22.

91. Cai X, et al. Long noncoding RNA Taurine-Upregulated gene 1 knockdown protects Cardiomyocytes against hypoxia/Reoxygenationinduced injury through regulating miR-532-5p/Sox8 Axis. J Cardiovasc Pharmacol. 2020;76(5):556-63.

92. Wang Z, et al. MicroRNA-326 prevents sepsis-induced acute lung injury via targeting TLR4. Free Radic Res. 2020;54(6):408-18.

93. Cui $\mathrm{Q}$, et al. Modulating ROS to overcome multidrug resistance in cancer. Drug Resist Updates. 2018;41:1-25.

94. Steinbichler T, et al. Therapy resistance mediated by cancer stem cells. Semin Cancer Biol. 2018;53:156-67.

95. Sun $X$, et al. MicroRNA-223 increases the sensitivity of triple-negative breast cancer stem cells to TRAIL-induced apoptosis by targeting HAX1. PLoS One. 2016;11(9):e0162754.

96. Yang W, et al. MicroRNA-153/Nrf-2/GPx1 pathway regulates radiosensitivity and stemness of glioma stem cells via reactive oxygen species. Oncotarget. 2015;6(26):22006-27.

97. Yang W, et al. Knockdown of miR-210 decreases hypoxic glioma stem cells stemness and radioresistance. Exp Cell Res. 2014;326(1):22-35.

98. Ding K, et al. Effect of long non-coding RNA H19 on oxidative stress and chemotherapy resistance of CD133+ cancer stem cells via the MAPK ERK signaling pathway in hepatocellular carcinoma. Biochem Biophys Res Commun. 2018;502(2):194-201.

99. Serguienko A, et al. Metabolic reprogramming of metastatic breast cancer and melanoma by let-7a microRNA. Oncotarget. 2015;6(4):2451-65.

100. $\mathrm{Xu} Z$, et al. miR-17-3p Downregulates mitochondrial antioxidant enzymes and enhances the Radiosensitivity of prostate cancer cells. Mol Ther Nucleic Acids. 2018;13:64-77.

101. Benassi $B$, et al. USP2a alters chemotherapeutic response by modulating redox. Cell Death Dis. 2013;4:e812.

102. Huang $C$, et al. The effects of ultrasound exposure on P-glycoproteinmediated multidrug resistance in vitro and in vivo. J Exper Clin Cancer Res. 2018:37(1):232.

103. Li P, et al. Low-intensity ultrasound enhances the chemosensitivity of hepatocellular carcinoma cells to cisplatin via altering the miR-34a/cmet axis. Int J Mol Med. 2019;44(1):135-44.

104. Moreno Leon L, et al. The nuclear hypoxia-regulated NLUCAT1 long non-coding RNA contributes to an aggressive phenotype in lung adenocarcinoma through regulation of oxidative stress. Oncogene. 2019;38(46):7146-65.

105. Zhang N, et al. LncRNA LINC00963 promotes tumorigenesis and Radioresistance in breast cancer by sponging miR-324-3p and inducing ACK1 expression. Mol Ther Nucleic Acids. 2019;18:871-81.

106. Yang $Q$, et al. IncRNA SLC7A11-AS1 promotes Chemoresistance by blocking SCF $\beta$-TRCP-mediated degradation of NRF2 in pancreatic cancer. Mol Ther Nucleic Acids. 2020;19:974-85.

107. Geng J, Yang K. circCCND1 regulates oxidative stress and FGF9 to enhance Chemoresistance of non-small cell lung cancer via sponging miR-187-3p. DNA Cell Biol. 2021;40(5):675-82.

108. Zeng Z, et al. Hypoxic exosomal HIF-1a-stabilizing circZNF91 promotes chemoresistance of normoxic pancreatic cancer cells via enhancing glycolysis. Oncogene. 2021;40(36):5505-17.

109. Xian Z, et al. IncRNA UCA1 contributes to 5-fluorouracil resistance of colorectal cancer cells through miR-23b-3p/ZNF281 Axis. OncoTargets Ther. 2020;13:7571-83. 
110. Zhang $P$, et al. HER2A novel tumor suppressor ZBTB1 regulates tamoxifen resistance and aerobic glycolysis through suppressing expression in breast cancer. J Biol Chem. 2020;295(41):14140-52.

111. YiRen $\mathrm{H}$, et al. Long noncoding RNA MALAT1 regulates autophagy associated chemoresistance via miR-23b-3p sequestration in gastric cancer. Mol Cancer. 2017;16(1):174.

112. Liang $\mathrm{S}$, et al. Profiling of circular RNAs in age-related cataract reveals circZNF292 as an antioxidant by sponging miR-23b-3p. Aging. 2020;12(17):17271-87.

113. Pant K, et al. Butyrate induces ROS-mediated apoptosis by modulating miR-22/SIRT-1 pathway in hepatic cancer cells. Redox Biol. 2017:12:340-9.

114. Li X, Pathi SS, Safe S. Sulindac sulfide inhibits colon cancer cell growth and downregulates specificity protein transcription factors. BMC Cancer. 2015;15:974.

115. Pathi SS, et al. GT-094, a NO-NSAID, inhibits colon cancer cell growth by activation of a reactive oxygen species-microRNA-27a: ZBTB10-specificity protein pathway. Mol Cancer Res. 2011;9(2):195-202.

116. Jutooru I, et al. Mechanism of action of phenethylisothiocyanate and other reactive oxygen species-inducing anticancer agents. Mol Cell Biol. 2014;34(13):2382-95.

117. Huang $\mathrm{C}-\mathrm{H}$, et al. Quinacrine induces the apoptosis of human leukemia U937 cells through FOXP3/miR-183/ $\beta-T r C P / S P 1$ axis-mediated BAX upregulation. Toxicol Appl Pharmacol. 2017;334:35-46.

118. Chen $\mathrm{P}$, et al. Curcumin promotes osteosarcoma cell death by activating miR-125a/ERRa signal pathway. J Cell Biochem. 2017;118(1):74-81.

119. Yang J, et al. p53-p66(shc)/miR-21-Sod2 signaling is critical for the inhibitory effect of betulinic acid on hepatocellular carcinoma. Toxicol Lett. 2015;238(3):1-10.

120. Wu L, et al. Polygonatum odoratum lectin induces apoptosis and autophagy by regulation of microRNA-1290 and microRNA-15a-3p in human lung adenocarcinoma A549 cells. Int J Biol Macromol. 2016;85:217-26

121. Xie C, et al. FeS@BSA Nanoclusters to enable H2S-amplified ROS-based therapy with MRI guidance. Adv Sci. 2020;7(7):1903512.

122. Setyawati MI, Leong DT. Mesoporous silica nanoparticles as an Antitumoral-angiogenesis strategy. ACS Appl Mater Interfaces. 2017;9(8):6690-703.

123. Yousefi A-M, et al. ZnO/CNT@Fe3O4 induces ROS-mediated apoptosis in chronic myeloid leukemia (CML) cells: an emerging prospective for nanoparticles in leukemia treatment. Artif Cells Nanomed Biotechnol. 2020;48(1):735-45.

124. Kou L, et al. Ambidextrous approach to disrupt redox balance in tumor cells with increased ROS production and decreased GSH synthesis for cancer therapy. ACS Appl Mater Interfaces. 2019;11(30):26722-30.

125. Takagawa Y, et al. miR-1293, a candidate for miRNA-based cancer therapeutics, simultaneously targets BRD4 and the DNA repair pathway. Mol Ther. 2020;28(6):1494-505.

126. Segatto $M$, et al. BETs inhibition attenuates oxidative stress and preserves muscle integrity in Duchenne muscular dystrophy. Nat Commun. 2020;11(1):6108.

127. Bayraktar R, et al. Dual suppressive effect of miR-34a on the FOXM1/ eEF2-kinase Axis regulates triple-negative breast cancer growth and invasion. Clin Cancer Res. 2018;24(17):4225-41.

128. Rokavec $M$, et al. IL-6R/STAT3/miR-34a feedback loop promotes EMTmediated colorectal cancer invasion and metastasis. J Clin Invest. 2014;124(4):1853-67.

129. Zhou Y, et al. IGF2BP3 functions as a potential oncogene and is a crucial target of miR-34a in gastric carcinogenesis. Mol Cancer. 2017;16(1):77.

130. Deng $X$, et al. Hsa-miR-34a-5p reverses multidrug resistance in gastric cancer cells by targeting the $3^{\prime}$-UTR of SIRT1 and inhibiting its expression. Cell Signal. 2021;84:110016.

131. Li H, et al. Cisplatin-induced epigenetic activation of miR-34a sensitizes bladder cancer cells to chemotherapy. Mol Cancer. 2014;13:8.

132. LiY, et al. UTMD promoted local delivery of miR-34a-mimic for ovarian cancer therapy. Drug Delivery. 2021;28(1):1616-25.

133. Yin $\mathrm{H}$, et al. RNA micelles for the systemic delivery of anti-miRNA for cancer targeting and inhibition without ligand. ACS Nano. 2019;13(1):706-17.
134. Shu D, et al. Systemic delivery of anti-miRNA for suppression of triple negative breast cancer utilizing RNA nanotechnology. ACS Nano. 2015;9(10):9731-40

135. Kai A, et al. Down-regulation of TIMP2 by HIF-1a/miR-210/HIF-3a regulatory feedback circuit enhances cancer metastasis in hepatocellular carcinoma. Hepatology (Baltimore, Md). 2016;64(2):473-87.

136. Ren D, et al. Oncogenic miR-210-3p promotes prostate cancer cell EMT and bone metastasis via NF-KB signaling pathway. Mol Cancer. 2017;16(1):117.

137. Xie Y, et al. Cholangiocarcinoma therapy with nanoparticles that combine downregulation of MicroRNA-210 with inhibition of cancer cell invasiveness. Theranostics. 2018;8(16):4305-20.

138. Gong N, et al. Antisense oligonucleotide-conjugated nanostructuretargeting IncRNA MALAT1 inhibits Cancer metastasis. ACS Appl Mater Interfaces. 2019;11(1):37-42.

139. Stuchi $L$, et al. VEGFA and gene expression and regulation by MicroRNAs in thyroid papillary cancer and colloid goiter. Genes. 2020;11(9):954.

140. Chen J, et al. LINC00173.v1 promotes angiogenesis and progression of lung squamous cell carcinoma by sponging miR-511-5p to regulate VEGFA expression. Mol Cancer. 2020;19(1):98.

141. Pichler M, et al. Therapeutic potential of FLANC, a novel primate-specific long non-coding RNA in colorectal cancer. Gut. 2020;69(10):1818-31.

142. Kim S, et al. Targeted nanocomplex carrying siRNA against MALAT1 sensitizes glioblastoma to temozolomide. Nucleic Acids Res. 2018:46(3):1424-40.

143. Wang R, et al. EIF4A3-induced circular RNA MMP9 (circMMP9) acts as a sponge of miR-124 and promotes glioblastoma multiforme cell tumorigenesis. Mol Cancer. 2018;17(1):166.

144. Taniguchi $\mathrm{K}$, et al. MicroRNA-124 inhibits cancer cell growth through PTB1/PKM1/PKM2 feedback cascade in colorectal cancer. Cancer Lett. 2015;363(1):17-27.

145. Huang W, et al. circRNA circAF4 functions as an oncogene to regulate MLL-AF4 fusion protein expression and inhibit MLL leukemia progression. J Hematol Oncol. 2019;12(1):103.

146. Chen $Z$, et al. Circular RNA cia-MAF drives self-renewal and metastasis of liver tumor-initiating cells via transcription factor MAFF. J Clin Invest. 2021;131(19):e148020.

147. Massrieh W, Derjuga A, Blank V. Induction of endogenous Nrf2/small maf heterodimers by arsenic-mediated stress in placental choriocarcinoma cells. Antioxid Redox Signal. 2006:8:53-9.

148. Wang $L$, et al. Estrogen-induced circRNA, circPGR, functions as a ceRNA to promote estrogen receptor-positive breast cancer cell growth by regulating cell cycle-related genes. Theranostics. 2021;11(4):1732-52.

149. Jiang $H$, LV J. MicroRNA-301a-3p increases oxidative stress, inflammation and apoptosis in ox-LDL-induced HUVECs by targeting KLF7. Exper Ther Med. 2021:21(6):569.

150. Winkle M, et al. Noncoding RNA therapeutics - challenges and potentia solutions. Nat Rev Drug Discov. 2021;20(8):629-51.

151. Adi Harel S, et al. Reactivation of epigenetically silenced miR-512 and miR-373 sensitizes lung cancer cells to cisplatin and restricts tumor growth. Cell Death Differ. 2015;22(8):1328-40.

152. Voorhoeve $P$, et al. A genetic screen implicates miRNA-372 and miRNA-373 as oncogenes in testicular germ cell tumors. Cell. 2006;124(6):1169-81.

153. Chen C, et al. MicroRNA-155 regulates arsenite-induced malignant transformation by targeting Nrf2-mediated oxidative damage in human bronchial epithelial cells. Toxicol Lett. 2017;278:38-47.

154. Liu Q, et al. miR-155 regulates Glioma cells invasion and Chemosensitivity by p38 Isforms in vitro. J Cell Biochem. 2015;116(7):1213-21.

155. Seux M, et al. TP53INP1 decreases pancreatic cancer cell migration by regulating SPARC expression. Oncogene. 2011;30(27):3049-61.

156. Lin S, et al. miR-371/372/373Activation of the miRNA cluster enhances Oncogenicity and drug resistance in Oral carcinoma cells. Int J Mol Sci. 2020;21(24):9442

157. Wang $\mathrm{H}$, et al. MiR-371 promotes proliferation and metastasis in hepatocellular carcinoma by targeting PTEN. BMB Rep. 2019;52(5):312-7.

158. Sahu N, et al. Functional screening implicates miR-371-3p and peroxiredoxin 6 in reversible tolerance to cancer drugs. Nat Commun. 2016;7:12351. 
159. Reid G, et al. Restoring expression of miR-16: a novel approach to therapy for malignant pleural mesothelioma. Ann Oncol. 2013;24(12):3128-35.

160. Andriani F, et al. MiR-16 regulates the pro-tumorigenic potential of lung fibroblasts through the inhibition of HGF production in an FGFR-1- and MEK1-dependent manner. J Hematol Oncol. 2018;11(1):45.

161. Ni C, et al. Breast cancer-derived exosomes transmit IncRNA SNHG16 to induce CD73+ $\gamma \delta 1$ Treg cells. Signal Transduct Targeted Ther. 2020;5(1):41.

162. Sampath $\mathrm{D}$, et al. Histone deacetylases mediate the silencing of miR15a, miR-16, and miR-29b in chronic lymphocytic leukemia. Blood. 2012;119(5):1162-72.

163. Zhang J, Chen Y, Gao W. Puerarin protects against human retinal endothelial cells injury induced by high glucose via regulating miR16-5p/CASP1 axis. Gen Physiol Biophys. 2021;40(3):235-43.

164. van Zandwijk N, et al. Safety and activity of microRNA-loaded minicells in patients with recurrent malignant pleural mesothelioma: a firstin-man, phase 1, open-label, dose-escalation study. Lancet Oncol. 2017;18(10):1386-96.

165. Jiang K, et al. miR-155-5p promotes oxalate- and calcium-induced kidney oxidative stress injury by suppressing MGP expression. Oxidative Med Cell Longev. 2020;2020:5863617.

166. LiY, et al. Bmi-1-induced miR-27a and miR-155 promote tumor metastasis and chemoresistance by targeting RKIP in gastric cancer. Mol Cancer. 2020;19(1):109.

167. Wallace J, et al. miR-155 promotes FLT3-ITD-induced myeloproliferative disease through inhibition of the interferon response. Blood. 2017;129(23):3074-86

168. Chen Y, et al. Long non-coding RNAs: from disease code to drug role. Acta Pharm Sin B. 2021;11(2):340-54.

169. Gao Y, et al. Long non-coding RNA ASncmtRNA-2 is upregulated in diabetic kidneys and high glucose-treated mesangial cells. Exper Ther Med. 2017:13(2):581-7.

170. Fitzpatrick $C$, et al. Mitochondrial ncRNA targeting induces cell cycle arrest and tumor growth inhibition of MDA-MB-231 breast cancer cells through reduction of key cell cycle progression factors. Cell Death Dis. 2019;10(6):423.

171. Borgna $\mathrm{V}$, et al. Mitochondrial ASncmtRNA-1 and ASncmtRNA-2 as potent targets to inhibit tumor growth and metastasis in the RenCa murine renal adenocarcinoma model. Oncotarget. 2017:8(27):43692-708.

172. Dhawan MS, et al. Phase 1 study of ANDES-1537: a novel antisense oligonucleotide against non-coding mitochondrial DNA in advanced solid tumors. J Clin Oncol. 2018;36(15):2557.

173. Garufi A, et al. A ruthenium (II)-curcumin compound modulates NRF2 expression balancing the cancer cell death/survival outcome according to p53 status. J Exper Clin Cancer Res. 2020;39(1):122.

174. Pan J, et al. A negative feedback loop of H19/miR-675/NDR mediates therapeutic effect of cucurmin in the treatment of glioma. J Cell Physiol. 2020;235(3):2171-82.

175. Yoshida K, et al. Curcumin sensitizes pancreatic cancer cells to gemcitabine by attenuating PRC2 subunit EZH2, and the InCRNA PVT1 expression. Carcinogenesis. 2017:38(10):1036-46.

176. Wang $Q$, et al. Curcumin enhances the radiosensitivity in nasopharyngeal carcinoma cells involving the reversal of differentially expressed long non-coding RNAs. Int J Oncol. 2014;44(3):858-64.

177. Xu X, et al. Curcumin suppresses the malignancy of non-small cell lung cancer by modulating the circ-PRKCA/miR-384/ITGB1 pathway. Biomed Pharmacother= Biomedecine \& pharmacotherapie. 2021;138:111439.

178. Zhu D, et al. Curcumin enhances Radiosensitization of nasopharyngeal carcinoma via mediating regulation of tumor stem-like cells by a CircRNA network. J Cancer. 2020;11 (8):2360-70

179. Aggarwal B, Kumar A, Bharti A. Anticancer potential of curcumin: preclinical and clinical studies. Anticancer Res. 2003:23:363-98.

180. Morató $\mathrm{L}$, et al. Activation of sirtuin 1 as therapy for the peroxisomal disease adrenoleukodystrophy. Cell Death Differ. 2015;22(11):1742-53.
181. Geng W, et al. Resveratrol inhibits proliferation, migration and invasion of multiple myeloma cells via NEAT1-mediated Wnt/ $\beta$-catenin signaling pathway. Biomed Pharmacother= Biomedecine \& pharmacotherapie. 2018;107:484-94.

182. Nguyen A, et al. Results of a phase I pilot clinical trial examining the effect of plant-derived resveratrol and grape powder on Wnt pathway target gene expression in colonic mucosa and colon cancer. Cancer Manag Res. 2009;1:25-37.

\section{Publisher's Note}

Springer Nature remains neutral with regard to jurisdictional claims in published maps and institutional affiliations.

Ready to submit your research? Choose BMC and benefit from

- fast, convenient online submission

- thorough peer review by experienced researchers in your field

- rapid publication on acceptance

- support for research data, including large and complex data types

- gold Open Access which fosters wider collaboration and increased citations

- maximum visibility for your research: over $100 \mathrm{M}$ website views per year

At BMC, research is always in progress.

Learn more biomedcentral.com/submissions 